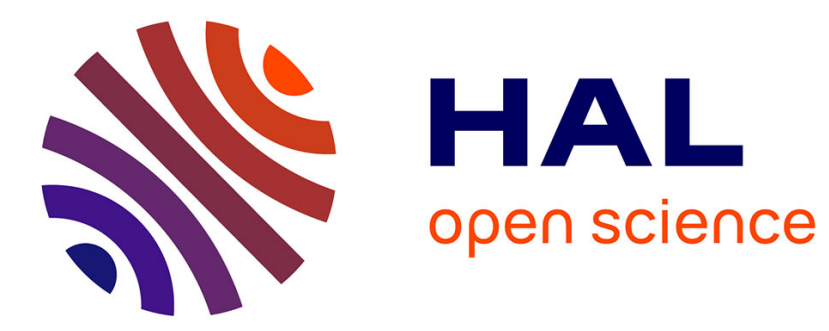

\title{
The magneto-hydrodynamic instabilities in rotating and precessing sheared flows: An asymptotic analysis
}

\author{
Abdelaziz Salhi, Thierry Lehner, Claude Cambon
}

\section{To cite this version:}

Abdelaziz Salhi, Thierry Lehner, Claude Cambon. The magneto-hydrodynamic instabilities in rotating and precessing sheared flows: An asymptotic analysis. Physical Review E : Statistical, Nonlinear, and Soft Matter Physics, 2010, 82, pp.016315. 10.1103/PhysRevE.82.016315 . hal-00566060

\section{HAL Id: hal-00566060 https://hal.science/hal-00566060}

Submitted on 3 Sep 2012

HAL is a multi-disciplinary open access archive for the deposit and dissemination of scientific research documents, whether they are published or not. The documents may come from teaching and research institutions in France or abroad, or from public or private research centers.
L'archive ouverte pluridisciplinaire HAL, est destinée au dépôt et à la diffusion de documents scientifiques de niveau recherche, publiés ou non, émanant des établissements d'enseignement et de recherche français ou étrangers, des laboratoires publics ou privés. 


\title{
The magneto-hydrodynamic instabilities in rotating and precessing sheared flows: An asymptotic analysis
}

\author{
A. Salhi ${ }^{1}$, T. Lehner ${ }^{2}$ \& C. Cambon ${ }^{3}$ \\ ${ }^{1}$ Département de Physique, Faculté des Sciences de Tunis, Tunisia. \\ ${ }^{2}$ Laboratoire LUTH, UMR 8102, CNRS, \\ Observatoire de Paris, 5 place de Janssen, 92195 Meudon. \\ ${ }^{3}$ Laboratoire de Mécanique des Fluides et d Acoustique, \\ Ecole Centrale de Lyon, UMR 5509, CNRS, \\ INSA, UCB, 69134 Ecully Cedex, France.
}




\begin{abstract}
Linear magneto-hydrodynamic instabilities are studied analytically in the case of unbounded inviscid and electrically conducting flows that are submitted to both rotation and precession with shear in an external magnetic field. For given rotation and precession the possible configurations of the shear and of the magnetic field and their interplay are imposed by the "admissibility" condition (i.e., the base flow must be a solution of the magnetohydrodynamic Euler equations): We show that an "admissible" basic magnetic field must align with the basic absolute vorticity. For these flows with elliptical streamlines due to precession we undertake an analytic stability analysis for the corresponding Floquet system, by using an asymptotic expansion into the small parameter $\varepsilon$ (ratio of precession to rotation frequencies) by a method first developed in the magneto-elliptical instabilities study by Lebovitz and Zweibel (Astrophys. J., 609, 301, 2004). The present stability analysis is performed into a suitable frame that is obtained by a systematic change of variables guided by symmetry and the existence of invariants of motion. The obtained Floquet system depends on three parameters: $\varepsilon, \eta$ (ratio of the cyclotron frequency to the rotation frequency) and $\chi=\cos \alpha, \alpha$ being a characteristic angle which, for circular streamlines, $\varepsilon=0$, identifies with the angle between the wave vector and the axis of the solid body rotation. We look at the various (centrifugal or precessional) resonant couplings between the three present modes: hydrodynamical (inertial), magnetic (Alfven) and mixed (magneto-inertial) modes by computing analytically to leading order in $\varepsilon$ the instabilities by estimating their threshold, growth rate and maximum growth rate and their bandwidths as function of $\varepsilon, \eta$ and $\chi$. We show that the subharmonic "magnetic" mode appear only for $\eta>\sqrt{5} / 2$ and at large $\eta(\gg 1)$ the maximal growth rate of both the "hydrodynamic" and "magnetic" modes approaches $\varepsilon / 2$, while the one of the subharmonic "mixed" mode approaches zero.
\end{abstract}




\section{INTRODUCTION}

Rotating and precessing flows are very common in Nature for example in celestial objects such as planets for which the combination of proper rotation and of gravitation lead to tidal forces making small elliptical deformations of the initial sphere. This may explain why both neutral and conducting fluids in such configurations have been considered in a lot of studies on theoretical and experimental grounds, in particular precessing flows for their importance in geophysics and in astrophysics, especially for the understanding of natural dynamos. Neutral fluids in rotating spheroids can lead in particular to both elliptical and centrifugal instabilities due to the possible coupling of the inertial waves that are excited by (fast) rotation and coupled by (slower) ellipticity or precession. Rossby waves can be also driven in rotating fluids in the presence of curved boundaries for example in oceans. In the case of the elliptical instability however earlier studies have been mainly motivated not by natural objects but by hydrodynamical considerations aimed at describing the evolution of vortices in a stretching field. The context of trailing vortices is still relevant, from the work

of Crow [1], but an important simplification consisted of disconnecting ellipticity from the mutual induction of adjacent counter-rotating vortices. For instance, the stability analysis of a single vortex flow with elliptical streamlines by Pierrehumbert [2] and by Bayly [3], using different methods, gave a new interest to this instability, whose a good review can be found in Kerswell [4]. It is perhaps useful to recall that Bayly used disturbances in terms of advected Fourier modes, with time-dependent wave vector, as in Rapid Distortion Theory (RDT hereinafter) for unbounded flows, since the same technique is used in the present paper and in many other mentioned here. RDT was introduced by Batchelor and Proudman [5] for disturbances to irrotational mean flows, but Moffatt [6] was probably the first to study the linear response of disturbances to a rotational flow (a pure plane shear), calculating a complete Green's function for individual disturbance modes. In addition, he proposed to coin "Kelvin mode" the Fourier mode with time-dependent wave vector, which derives from an eikonal equation. The reader is also referred to Cambon et al. [7] for generalization of this technique to base flows with hyperbolic, rectilinear and elliptical streamlines in a rotating frame.

Very recent developments on the elliptical flow instability in terms of classical normal mode analysis, including nonlinear evolution of the instabilities by going to mode couplings 
at higher order, can be found for example in Eloy et al.[8, 9] and Lacaze et al. [10] with both theoretical and experimental approaches. Its original result comes from the notice that a 2D flow with elliptical streamlines is found to be unstable to wave perturbations. This is in particular the case for the Kerswell flow ([11], KBF hereafter) under study here which is characterized by the following configuration: its base flow has an horizontal shear whose cross gradient is normal to both main (reference direction) and precession (here taken at right angle from main rotation) rotations axis, while the Mahalov base flow ([12] MBF hereafter) has instead a vertical shear with a cross gradient aligned with the main rotation. These flows can be subject to both elliptical and centrifugal instabilities, these denominations correspond to the coupling of the two inertial eigenmodes of the system such that their azimuthal numbers differ by $\Delta m=1$ for the centrifugal case and by $\Delta m=2$ for the elliptical case. A detailed comparison of the linear stage of the instability for these two flows, using RDT and therefore ignoring solid boundaries, has been done in a previous paper (Salhi \& Cambon [13]) without magnetic field. For the centrifugal instability a detailed theoretical and experimental study can be found in Gans [14]. These instabilities are still actively studied also for their nonlinear saturation and compared with experiments conducted on purpose (see for example in a cylinder the recent works of Meunier et al. [15], and of Lehner et al. [16]).

In the presence of magnetic fields various magnetohydrodynamical and here magnetoCoriolis waves $([17,19])$ can be excited, and magneto-Archimedes- Coriolis (MAC) waves as well in the presence of an additional density stratification ([20,21]). In the case of accretion disk in astrophysics it is widely believed that the angular momentum transport might be driven by turbulence and would be very improbable without the presence of the magnetic field (see for example Balbus and Hawley [22]). A close topic concerns the description of the magneto-rotational instability and of its saturation for accretion disk but in the presence of differential rotation (for example a Keplerian one), it is still a very active area of research ([23-25]) in various domains of physics.

For the specific case of dynamo and precession, Gans [26] undertook a hydromagnetic study in liquid sodium in precession where he observed an amplification of an initial feeding magnetic field by a factor of 3 (see also Lacaze et al. [10]).

It has been pointed out that buoyancy can trigger planetary and stellar dynamos. Bullard [27] and after him, Malkus [28] have suggested on purely energetic grounds that precession 


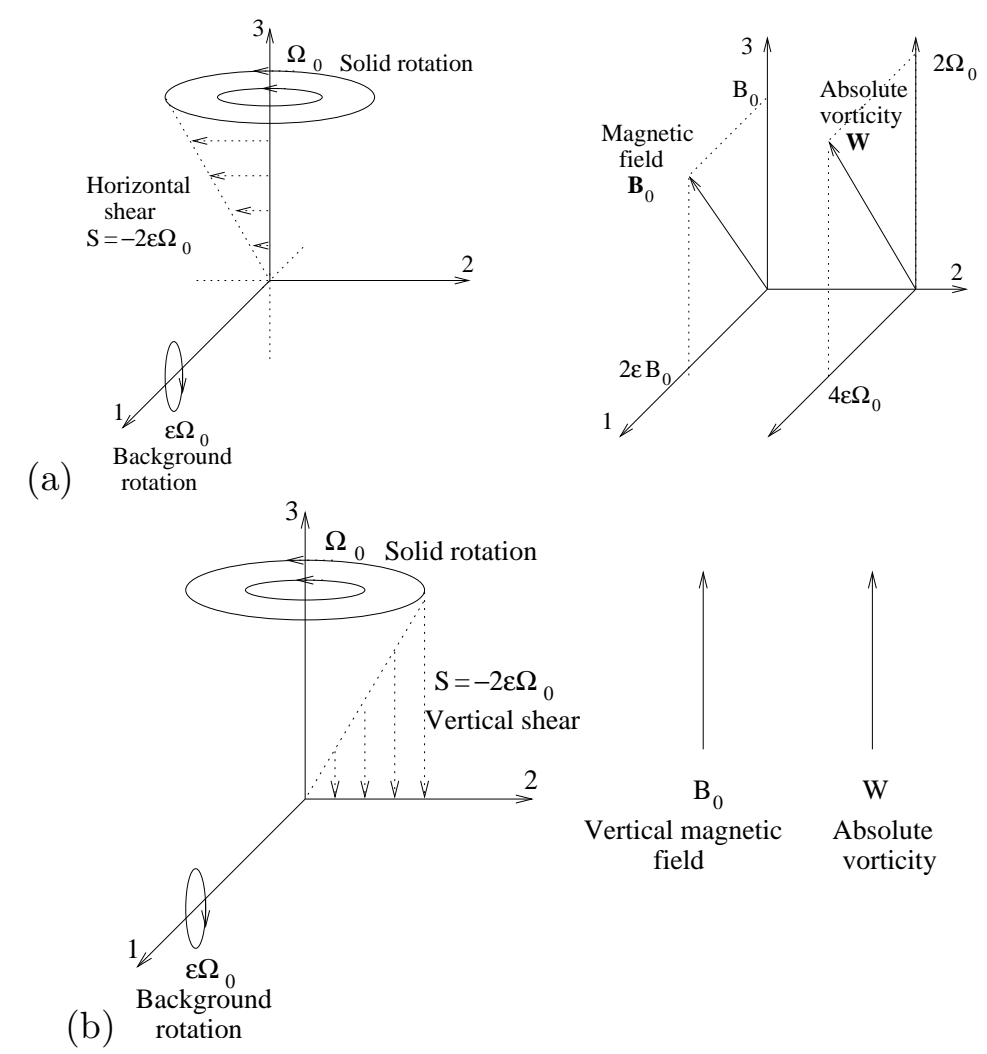

FIG. 1: Rotating precessing shear flows in magnetic fields. (a) The Kerswell base flow (KBF): Solid body rotation (with rate $\Omega_{0}$ ), background rotation (with rate $2 \varepsilon \Omega_{0}$ ), horizontal shear $\left(U_{i}=S x_{3} \delta_{i 2}\right.$, with rate $\left.S=-2 \varepsilon \Omega_{0}\right)$, external magnetic fied $\left(B_{i}=2 \varepsilon B_{0} \delta_{i 1}+B_{0} \delta_{i 3}\right)$. (b) The Mahalov base flow $(\mathrm{MBF})$ : Solid body rotation (with rate $\Omega_{0}$ ), background rotation (with rate $2 \varepsilon \Omega_{0}$ ), vertical shear $\left(U_{i}=S x_{2} \delta_{i 3}\right.$, with rate $\left.S=-2 \varepsilon \Omega_{0}\right)$, external magnetic field $\left(B_{i}=B_{0} \delta_{i 3}\right)$.

can be a viable driving mechanism for the geodynamo. For the special case of a precessing sphere, it is known that differential rotation which is a fundamental feature for powering dynamo action, may be induced by a balance between viscosity and nonlinear effects (Zhang et al. [29]). However, it remains an open question to know whether precession driven flow has a suitable structure for magnetic field generation (Tilgner [30]). But, recently, Wu and Roberts [31] have claimed that they have obtained numerically dynamo action in precessing spheroids. This result stimulates new interests for this topic in general but also for finding driving mechanisms suitable for laboratory experiments scale dynamo which still remains a challenge (Gailitis [32]). This type of forcing of an inertial $m=1$ mode by precession is met frequently in astrophysical objects including the Earth $([23,24,26])$. 
We present a theoretical asymptotic linear analysis of the magnetohydrodynamical instabilities that might be present in the case of the two quoted unbounded base flows: KBF and MBF flows. These base flows are with elliptic streamlines due to the action of the precession (along an horizontal axis, say $x_{2}$ ) on the circular streamlines of the vertical solid rotation (see figure 1). Similarities between precessing sheared flows and two-dimensional flows with elliptical streamlines (see e.g., [2, 3, 33, 34]) has been considered by $\operatorname{Kerswell~}([4,11]$, then by [13]), and are investigated in the present study accounting for the effect of an external magnetic field. As we will show here, the "admissibility" condition [33] with MHD coupling, which means that the base flow must be a solution of the magnetohydrodynamical Euler equations, requires that the basic magnetic field aligns with the basic absolute vorticity: For the MBF case, the magnetic field is then vertical, while for the KBF case, it has, in addition to a vertical component $B_{0}$, an horizontal one, $2 \varepsilon B_{0}$, (along the rotation axis of the background rotation).

Both Euler equations and induction equation are linearized. In addition to the Poincaré parameter $\varepsilon$, the magnetic parameter $\eta$ is the crucial parameter for MHD coupling. It is defined here as $\eta=V_{a} k / \Omega_{0}$, where $V_{a}$ is the Alfvén velocity, $\Omega_{0}$ is the angular velocity and $k$ is a wave number further specified in Eq. (17).It is a reciprocal Alfvén number of a sort, and it corresponds to the Lehnert number [18] if $k$ is replaced by the inverse of a typical lengthscale.

Disturbances in terms of Kelvin (i.e. base-flow advected Fourier) modes are governed by a Green's function [6, 7, 13], which reduces to a Floquet system, given the time-periodicity of the wave vector induced by close elliptical streamlines. This Floquet system is then analyzed at leading order of the precession parameter $\varepsilon$ by using the method first introduced in the study of magnetoelliptical instabilities by Lebovitz and Zweibel [35] and also used by Mizerski \& Bajer [36] to account for the effect of the Coriolis force on these instabilities.

In the first part (section 2) we set our formulation recalling first the basic equations we are dealing with, together with the three selected basic flows. Then we look at the 3D linearized perturbations around these base flows leading to a Floquet system, also by investigating useful special cases. In section 3 we perform the asymptotic expansion into the small precession parameter, by making first a convenient change of variables in order to simplify the (4-4) Floquet system and we compare it with the one derived in section 2 in the case of magneto-elliptical instabilities. We recover also the growth rate for the 
pure centrifugal case without the B field. Then we introduce in section 4 the sub-harmonic resonances arising from the solid body rotation. We put aside detailed computations that are given in appendices. Section 5 contains our discussion of results, and Section 6 is devoted to conclusions.

\section{FORMULATION}

The fluid is assumed inviscid and perfectly conducting. The equations describing the evolution of the velocity field $\tilde{\boldsymbol{u}}$ and the magnetic field $\tilde{\mathbf{b}}$ are the Navier-Stokes equations with the Lorentz and Coriolis forces and the induction equation,

$$
\begin{gathered}
\nabla \cdot \tilde{\boldsymbol{u}}=0 \\
\frac{\partial \tilde{\boldsymbol{u}}}{\partial t}+(\tilde{\boldsymbol{u}} \cdot \nabla) \tilde{\boldsymbol{u}}=-\frac{1}{\rho} \nabla \tilde{p}-2 \boldsymbol{\Omega} \times \tilde{\boldsymbol{u}}+\frac{1}{\rho}(\tilde{\boldsymbol{j}} \times \tilde{\boldsymbol{b}}) \\
\frac{\partial \tilde{\boldsymbol{b}}}{\partial t}+(\tilde{\boldsymbol{u}} \cdot \nabla) \tilde{\boldsymbol{b}}=(\tilde{\boldsymbol{b}} \cdot \nabla) \tilde{\boldsymbol{u}}
\end{gathered}
$$

where $\tilde{p}$ is the pressure modified by the centrifugal potential $(1 / 2) \rho(\boldsymbol{\Omega} \times \boldsymbol{x})^{2}, \rho$ is the fluid

density and $\tilde{j}$ is the density of the electric current. By neglecting the displacement current, the Maxwell laws take the form

$$
\nabla \cdot \tilde{\boldsymbol{b}}=0, \quad \tilde{\boldsymbol{j}}=\frac{1}{\mu_{0}} \nabla \times \tilde{\boldsymbol{b}}
$$

where $\mu_{0}$ denotes the magnetic permeability.

\subsection{Relevant neutral wave modes}

The MHD flow is subject to instabilities in the presence of ellipticity and/or precession. The neutral wave modes, however, that appear with uniform external magnetic field and solid body rotation with purely circular streamlines, are essential for a subsequent understanding of the development of instabilities, especially in the linear limit.

The equations above are linearized around a uniform $\boldsymbol{B}_{0}$ magnetic fluid, with no velocity base flow except the Coriolis force already present in eq. 2 .

As for many wave motions, a single equation can be found for the pressure disturbance $p$

$$
\frac{\partial^{4}}{\partial t^{4}} \nabla^{2} \tilde{p}-2\left(\boldsymbol{V}_{a} \nabla\right)^{2} \frac{\partial^{2}}{\partial t^{2}} \nabla^{2} \tilde{p}+4(\boldsymbol{\Omega} \nabla)^{2} \frac{\partial^{2}}{\partial t^{2}} \tilde{p}+\left(\boldsymbol{V}_{a} \nabla\right)^{4} \nabla^{2} p=0,
$$


introducing the Alvèn velocity

$$
\boldsymbol{V}_{a}=\frac{\boldsymbol{B}_{0}}{\sqrt{\rho \mu_{0}}}
$$

for dimensional convenience.

The dispersion relation is immediately recovered for wave-like solutions of the equation above, $p \sim \hat{p} \exp (\imath(\boldsymbol{k} \cdot \boldsymbol{x}-\omega t))$, as

$$
\omega^{4}-\left(\omega_{i}^{2}+2 \omega_{a}^{2}\right) \omega^{2}+\omega_{a}^{4}=0
$$

This equation involves the two basic dispersion laws, $\omega_{i}$ for inertial waves and $\omega_{a}$ for Alfvén waves, or

$$
\omega_{i}= \pm \frac{2 \Omega \cdot \boldsymbol{k}}{k} \text { and } \omega_{a}= \pm \boldsymbol{V}_{a} \cdot \boldsymbol{k}
$$

The solution of the dispersion relationship is finally found as

$$
\omega^{2}=\frac{1}{2} \omega_{i}^{2}+\omega_{a}^{2} \pm \sqrt{\left(\frac{1}{2} \omega_{i}^{2}+\omega_{a}^{2}\right)^{2}-\omega_{a}^{4}} .
$$

Without rotation, the case of pure Alfvén wave is recovered with $\omega^{2}=\omega_{a}^{2}$. Without external magnetic field, the two solutions $\omega=0$ (sign minus) and $\omega^{2}=\omega_{i}^{2}$ (sign plus) mean that the magnetic field is not affected by waves, whereas the hydrodynamic field is affected by pure inertial waves. When rotation and external magnetic field are simultaneously present, inertia-Alfvén waves do affect "mixed" magneto-hydrodynamic modes. A Poincaré-type equation for the pressure fluctuation can be found in the precessing case as well, but it would be much more complicated than Eq. (5). This equation (5) accounts for rotation through the Coriolis force, which corresponds only to the precession rotation in what follows, but without the effect of the main solid body rotation and related additional shear flow, forming the following base flow.

\subsection{Base flow}

The base flow considered in the present study corresponds to a vertical $\left(x_{3}\right)$ solid body rotation viewed in a rotating frame about the $x_{1}$ axis with additional plane shear in an external magnetic field,

$$
\boldsymbol{U}=\mathbf{A} \cdot \boldsymbol{x}, \quad \boldsymbol{\Omega}=\left[\varepsilon \Omega_{0}, 0,0\right]^{T}, \quad \boldsymbol{B}_{0}=\left[B_{01}, B_{02}, B_{03}\right]^{T}
$$


where

$$
\mathbf{A}=\Omega_{0}\left[\begin{array}{ccc}
0 & -1 & 0 \\
1 & 0 & -2 \varepsilon \\
0 & 0 & 0
\end{array}\right] \quad \text { or } \quad \mathbf{A}=\Omega_{0}\left[\begin{array}{ccc}
0 & -1 & 0 \\
1 & 0 & 0 \\
0 & -2 \varepsilon & 0
\end{array}\right]
$$

and $B_{0 j}=$ constant $(j=1,2,3)$ is not a priori specified. Here, $T$ denotes transpose.

The presence of the plane shear with rate $-2 \varepsilon \Omega_{0}$ in each one of these two base flows allows to ensure the admissibility conditions, previously mentioned (see e.g. Craik [33]). Physically, this additional plane shear, which results from the precession, was found in previous experimental studies (e.g., Wiener et al. [37], Lehner et al. [16]). More precisely, Salhi and Cambon [13] have shown how the prescribed shear exactly balances the gyroscopic torque, which results from the misalignment of main rotation axis and precessing rotation axis, in order to satisfy the equation for absolute vorticity.

The main difference between these two base flows is the cross-gradient direction of the plane shear: For the base flow described by the first relation in (10) it is horizontal, while for the base flow described by the first relation in (10) it is vertical. As in the study by Salhi \& Cambon [13], these base flows are referred to as the Kerswell base flow [11] (KBF) and the Mahalov base flow [12] (MBF), respectively. It should be noted that, for sufficiently small precessing parameter $(\varepsilon \ll 1)$, both flow cases $(\mathrm{KBF}$ and $\mathrm{MBF})$ can be deduced from the Poincaré's basic state of precessing spheroidal container (see [11, 13]).

Now, admissibility conditions are applied to the basic magnetic field. Because $\boldsymbol{B}_{0}$ is constant, the induction equation for the basic magnetic field reduces to $\mathbf{A} \cdot \boldsymbol{B}_{0}=\mathbf{0}$, and remains similar to the equation for the basic absolute vorticity $\boldsymbol{W}=\nabla \times \boldsymbol{U}+2 \boldsymbol{\Omega}$,

$$
\mathrm{A} \cdot \boldsymbol{W}=\mathbf{0}
$$

This implies that $\boldsymbol{B}_{0}$ aligns with $\boldsymbol{W}$. For the KBF case where the matrix $\mathbf{A}$ is described by the relation (10), the induction equation reduces to

$$
\left[\begin{array}{ccc}
0 & -1 & 0 \\
1 & 0 & -2 \varepsilon \\
0 & 0 & 0
\end{array}\right] \cdot\left[\begin{array}{c}
B_{01} \\
B_{02} \\
B_{03}
\end{array}\right]=\left[\begin{array}{l}
0 \\
0 \\
0
\end{array}\right]
$$

So that

$$
\boldsymbol{B}_{0}=B_{0}[2 \varepsilon, 0,1]^{T}, \quad \boldsymbol{W}=2 \Omega_{0}[2 \varepsilon, 0,1]^{T}
$$


It appears that the basic magnetic field has an horizontal component $\left(2 \varepsilon B_{0}\right)$ that can be seen as generated by the interaction between the horizontal plane shear and the vertical magnetic field. For the MBF case where the matrix $\mathbf{A}$ is described by the second relation in (10), the induction equation implies that $\boldsymbol{B}_{0}$ must align with the vertical axis,

$$
\boldsymbol{B}_{0}=B_{0}[0,0,1]^{T}, \quad \boldsymbol{W}=2 \Omega_{0}[0,0,1]^{T} .
$$

As it can be seen $\|\boldsymbol{W}\|=2 \Omega_{0}$ and $\left\|\boldsymbol{B}_{0}\right\|=B_{0}$ for the MBF and $\|\boldsymbol{W}\|=2 \Omega_{0} \sqrt{1+4 \varepsilon^{2}}$ and $\left\|\boldsymbol{B}_{0}\right\|=B_{0} \sqrt{1+4 \varepsilon^{2}}$ for the KBF. Obviously, without background rotation $(\varepsilon=0)$, both the flow cases reduce to a solid body rotation in a vertical magnetic field.

On the other hand, it should be informative for comparison to consider the "classical" case with elliptical streamlines studied by several authors (e.g., [3, 4, 7, 33-35]),

$$
\boldsymbol{U}=\Omega_{0}\left(-E x_{2}, E^{-1} x_{1}, 0\right)^{T} .
$$

In that case, the induction equation implies that $\boldsymbol{B}_{0}$ aligns with the vertical axis (see [35]).

\subsection{Three-dimensional perturbations}

We consider three-dimensional disturbances to the above basic precessing rotating flows in the form of single plane waves with a time-dependent wave vector,

$$
\left[\begin{array}{c}
\boldsymbol{u}(\boldsymbol{x}, t) \\
\frac{1}{\rho} p(\boldsymbol{x}, t) \\
\frac{1}{\sqrt{\rho \mu_{0}}} \boldsymbol{b}(\boldsymbol{x}, t)
\end{array}\right]=\left[\begin{array}{c}
\hat{\boldsymbol{u}}(\boldsymbol{k}, t) \\
\hat{p}(\boldsymbol{k}, t) \\
\hat{\boldsymbol{b}}(\boldsymbol{k}, t)
\end{array}\right] \exp [\imath \boldsymbol{k}(t) \cdot \boldsymbol{x}]
$$

in which $\hat{\boldsymbol{b}}$ has the same dimension as the velocity modes $\hat{u}_{i}(\boldsymbol{k}, t)$. The admissible base flow is shown to be compatible with the wavelike form for the disturbance flow, and the superposition of both is called "a class of exact solutions" for Euler equations (see Craik [33]). This is nothing other than a formal rediscovery of RDT, mentioned in introduction, but one in which nonlinearity is rigorously excluded in the equations for the disturbance flow: Only single-mode perturbation is considered and nonlinearity is then zero (at least for divergence-free flows.) 
The wave vector $\boldsymbol{k}$ satisfies the eikonal-type equation: $d \boldsymbol{k} / d t=-\mathbf{A}^{T} \cdot \boldsymbol{k}$. For the KBF case, it reduces to

$$
\frac{d}{d t}\left[k_{1}, k_{2}, k_{3}\right]^{T}=\Omega_{0}\left[-k_{2}, k_{1}, 2 \varepsilon k_{2}\right]^{T}
$$

with solution (see [11, 13]),

$$
\begin{gathered}
{\left[k_{1}, k_{2}, k_{3}\right]^{T}=\left[k_{p} \cos \tau, k_{p} \sin \tau, k_{0}-2 \varepsilon k_{p} \cos \tau\right]^{T}} \\
\tau=\Omega_{0} t+\arctan \left(K_{2} / K_{1}\right), \quad k_{0}=k_{3}+2 \varepsilon k_{1}=K_{3}+2 \varepsilon K_{1}, \\
k_{p}=\sqrt{k_{1}^{2}+k_{2}^{2}}=\sqrt{K_{1}^{2}+K_{2}^{2}} .
\end{gathered}
$$

Here, the capital letter $\boldsymbol{K}$ denotes initial value (at $t=0$ ). For the MBF, the eikonal-type equation reduces to

$$
\frac{d}{d t}\left[k_{1}, k_{2}, k_{3}\right]^{T}=\Omega_{0}\left[-k_{2}, k_{1}+2 \varepsilon k_{3}, 0\right]^{T}
$$

with solution [13],

$$
\begin{gathered}
{\left[k_{1}, k_{2}, k_{3}\right]^{T}=\left[-2 \varepsilon k_{0}+k_{p} \cos \tau, k_{p} \sin \tau, k_{0}\right]^{T},} \\
\tau=\Omega_{0} t+\arctan \left(K_{2} /\left(K_{1}+2 \varepsilon K_{3}\right)\right), \quad k_{0}=K_{3}=k_{3}, \\
k_{p}=\sqrt{\left(k_{1}+2 \varepsilon k_{3}\right)^{2}+k_{2}^{2}}=\sqrt{\left(K_{1}+2 \varepsilon K_{3}\right)^{2}+K_{2}^{2}} .
\end{gathered}
$$

For both flow cases (KBF and $\mathrm{MBF}$ ), the characteristic lines (or "trajectories" in wavespace) exhibit two invariants, namely $k_{0}$ and $k_{p}$ which define ellipses in wave-space [13]. As will be shown later, the stability problem for both flow cases depends on three parameters: The precessing parameter $\varepsilon$, the magnetic parameter $\eta$ and the characteristic angle $\alpha$ such that

$$
\eta=\frac{B_{0}}{\Omega_{0} \sqrt{\rho \mu_{0}}} \sqrt{k_{0}^{2}+k_{p}^{2}}, \quad \chi \equiv \cos \alpha=\frac{k_{0}}{\sqrt{k_{0}^{2}+k_{p}^{2}}} .
$$

The parameter $\eta$ is of the form $\eta=V_{a} k / \Omega_{0}$, in terms of the Alfvén velocity $V_{a}$ (Eq. (6)), and appears as a reciprocal Alfvén number of a sort, with a close linkage to the Lehnert number, as mentioned in introduction. When $\varepsilon=0$ (i.e., circular streamlines), the wavenumber $\sqrt{k_{0}^{2}+k_{p}^{2}}$ reduces to the modulus of the wave vector, and the angle $\alpha$ identifies with the polar angle (i.e., the angle between the wave vector and the vertical axis). 
We note that for the case with elliptical streamlines (given by equation (13)), the stability problem depends on the parameters $\varepsilon, \chi$ and $\eta$ such that

$$
\varepsilon=\frac{1}{2}\left(E-E^{-1}\right)
$$

while $\chi \equiv \cos \alpha$ and $\eta$ are described by (17) with

$$
\begin{gathered}
k_{0}=k_{3}=K_{3}, \quad k_{p}^{2}=k_{1}^{2}+E^{-2} k_{2}^{2}=K_{1}^{2}+E^{-2} K_{2}^{2}, \\
k_{1}=k_{p} \cos \tau, \quad k_{2}=k_{p} E \sin \tau=k_{p}\left(\varepsilon+\sqrt{1+\varepsilon^{2}}\right) \sin \tau,
\end{gathered}
$$

and

$$
\tau=\Omega_{0} t+\arctan \left(K_{2} /\left(E K_{1}\right)\right) .
$$

The equations for the disturbances

In view of the eikonal equation, the substitution of the form (14) into the equations for the perturbations derived from equations (1)-(4) yields,

$$
\begin{gathered}
\frac{d \hat{u}_{i}}{d t}+\left(A_{i j}+2 \varepsilon \Omega_{0} e_{i 1 j}\right) \hat{u}_{j}=-\imath \hat{p} k_{i}+\frac{\imath}{\sqrt{\rho \mu_{0}}}\left[(\boldsymbol{k} \times \hat{\boldsymbol{b}}) \times \boldsymbol{B}_{0}\right]_{i}, \\
\frac{d \hat{b}_{i}}{d t}-A_{i j} \hat{b}_{j}=\frac{\imath}{\sqrt{\rho \mu_{0}}}\left(B_{0 j} k_{j}\right) \hat{u}_{i}, \\
\boldsymbol{k} \cdot \hat{\boldsymbol{u}}=0, \quad \boldsymbol{k} \cdot \hat{\boldsymbol{b}}=0,
\end{gathered}
$$

where $\delta_{i j}$ is the Kronecker delta and $e_{i j k}$ is the permutation tensor. The pressure term is solved in order to satisfy the incompressibility constraint,

$$
\hat{p}=\hat{p}_{h}+\hat{p}_{m}=2 \imath \frac{k_{m}}{k^{2}}\left(A_{m n}+\Omega_{0} \varepsilon e_{m 1 n}\right) \hat{u}_{n}+\frac{1}{\sqrt{\rho \mu_{0}}} \frac{k_{m}}{k^{2}}\left[(\boldsymbol{k} \times \hat{\boldsymbol{b}}) \times \boldsymbol{B}_{0}\right]_{m},
$$

in which the first term $\hat{p}_{h}$ corresponds to the hydrodynamic part, while the second one $\hat{p}_{m}$ corresponds to the magnetic part,

$$
\hat{p}_{m}=-B_{0}\left(\hat{b}_{3}+2 \varepsilon \hat{b}_{1}\right) / \sqrt{\rho \mu_{0}}
$$

for the $\mathrm{KBF}$ case and

$$
\hat{p}_{m}=-B_{0} \hat{b}_{3} / \sqrt{\rho \mu_{0}}
$$


for the MBF case. The linear differential system for $\hat{\boldsymbol{u}}=\left(\hat{u}_{1}, \hat{u}_{2}, \hat{u}_{3}\right)^{T}$ and $\hat{\boldsymbol{b}}=\left(\hat{b}_{1}, \hat{b}_{2}, \hat{b}_{3}\right)^{T}$, is determined by substituting (22) into (19),

$$
\begin{gathered}
\frac{d \hat{u}_{1}}{d \tau}=2 \frac{k_{1} k_{2}}{k^{2}} \hat{u}_{1}+\left(1-2 \frac{k_{1}^{2}}{k^{2}}+2 \varepsilon \frac{k_{1} k_{3}}{k^{2}}\right) \hat{u}_{2}-6 \varepsilon \frac{k_{1} k_{2}}{k^{2}} \hat{u}_{3}+\imath \eta \chi \hat{b}_{1}, \\
\frac{d \hat{u}_{2}}{d \tau}=-\left(1-2 \frac{k_{2}^{2}}{k^{2}}\right) \hat{u}_{1}-2\left(\frac{k_{1} k_{2}}{k^{2}}-\varepsilon \frac{k_{2} k_{3}}{k^{2}}\right) \hat{u}_{2}+2 \varepsilon\left(2-3 \frac{k_{2}^{2}}{k^{2}}\right) \hat{u}_{3}+\imath \eta \chi \hat{b}_{2}, \\
\frac{d \hat{u}_{3}}{d \tau}=2 \frac{k_{2} k_{3}}{k^{2}} \hat{u}_{1}-\left[2 \frac{k_{1} k_{3}}{k^{2}}+2 \varepsilon\left(1-\frac{k_{3}^{2}}{k^{2}}\right)\right] \hat{u}_{2}-6 \varepsilon \frac{k_{2} k_{3}}{k^{2}} \hat{u}_{3}+\imath \eta \chi \hat{b}_{3}, \\
\frac{d \hat{b}_{1}}{d \tau}=-\hat{b}_{2}+\imath \chi \eta \hat{u}_{1}, \quad \frac{d \hat{b}_{2}}{d \tau}=b_{1}-2 \varepsilon \hat{b}_{3}+\imath \eta \chi \hat{u}_{2}, \quad \frac{d \hat{b}_{3}}{d \tau}=\imath \eta \chi \hat{u}_{3} .
\end{gathered}
$$

for the KBF case and

$$
\begin{gathered}
\frac{d \hat{u}_{1}}{d \tau}=2 \frac{k_{1} k_{2}}{k^{2}} \hat{u}_{1}+\left(1-2 \frac{k_{1}^{2}}{k^{2}}-2 \varepsilon \frac{k_{1} k_{3}}{k^{2}}\right) \hat{u}_{2}-2 \varepsilon \frac{k_{1} k_{2}}{k^{2}} \hat{u}_{3}+\imath \eta \chi \hat{b}_{1}, \\
\frac{d \hat{u}_{2}}{d \tau}=\left(-1+2 \frac{k_{2}^{2}}{k^{2}}\right) \hat{u}_{1}-\left(2 \frac{k_{1} k_{2}}{k^{2}}+2 \varepsilon \frac{k_{2} k_{3}}{k^{2}}\right) \hat{u}_{2}+2 \varepsilon\left(1-\frac{k_{2}^{2}}{k^{2}}\right) \hat{u}_{3}+\imath \eta \chi \hat{b}_{2}, \\
\frac{d \hat{u}_{3}}{d \tau}=2 \frac{k_{2} k_{3}}{k^{2}} \hat{u}_{1}-2\left(\frac{k_{1} k_{3}}{k^{2}}+\varepsilon \frac{k_{3}^{2}}{k^{2}}\right) \hat{u}_{2}-2 \varepsilon \frac{k_{2} k_{3}}{k^{2}} \hat{u}_{3}+\imath \eta \chi \hat{b}_{3}, \\
\frac{d \hat{b}_{1}}{d \tau}=-\hat{b}_{2}+\imath \eta \chi \hat{u}_{1}, \quad \frac{d \hat{b}_{2}}{d \tau}=\hat{b}_{1}+\imath \eta \chi \hat{u}_{2}, \quad \frac{d \hat{b}_{3}}{d \tau}=-2 \varepsilon \hat{b}_{2}+\imath \eta \chi \hat{u}_{3}
\end{gathered}
$$

for the MBF case. Because the wave vector is time-periodic (with period $2 \pi$ ), (23) and (24) are Floquet systems.

\subsection{The limit cases, $k_{p}=0$ and $k_{0}=0$}

We now examine the stability of the above Floquet systems at the limit cases, $k_{p}=0$ and $k_{0}=0$.

The KBF case

If $k_{p}=\sqrt{k_{1}^{2}+k_{2}^{2}}=0$, then $k_{1}=0$ and $k_{2}=0$, and due to the fact that both $\boldsymbol{u}$ and $\boldsymbol{b}$ are solenoidal, one obtains $k_{3} \hat{u}_{3}=0$ and $k_{3} \hat{b}_{3}=0$. Because the case where $\boldsymbol{k}=(0,0,0)^{T}$ (for which the perturbation is uniform with respect to the space coordinates) is not considered 
here, the disturbances associated to $k_{p}=0$ are two-dimensional lying to the horizontal plane. In that case, the Floquet system (23) reduces to

$$
\begin{aligned}
& \frac{d \hat{u}_{1}}{d \tau}=\hat{u}_{2}+\imath \eta \hat{b}_{1}, \quad \frac{d \hat{u}_{2}}{d \tau}=-\hat{u}_{1}+\imath \eta \hat{b}_{2}, \\
& \frac{d \hat{b}_{1}}{d \tau}=-\hat{b}_{2}+\imath \eta \hat{u}_{1}, \quad \frac{d \hat{b}_{2}}{d \tau}=\hat{b}_{1}+\imath \eta \hat{u}_{2},
\end{aligned}
$$

with eigenvalues $\lambda_{1,2}=\imath \sqrt{1+\eta^{2}}$ and $\lambda_{3,4}=-\imath \sqrt{1+\eta^{2}}$, indicating stability.

When $k_{0}=k_{3}+2 \varepsilon k_{1}=0$ and $|\eta|<+\infty$, so that $\eta \chi=0$, the Floquet system (31) can be transformed as

$$
\begin{gathered}
\frac{d}{d \tau}\left[k_{1} \hat{u}_{2}-k_{2} \hat{u}_{1}\right]=0, \quad \frac{d}{d \tau}\left[k^{2} \hat{u}_{3}-2 \varepsilon k_{2}\left(k_{1} \hat{u}_{2}-k_{2} \hat{u}_{1}\right)\right]=0, \\
\frac{d}{d \tau}\left[k_{1} \hat{b}_{2}-k_{2}\left(\hat{b}_{1}-\varepsilon \hat{b}_{3}\right)\right]=0, \quad \frac{d \hat{b}_{3}}{d \tau}=0
\end{gathered}
$$

indicating that, at $k_{0}=0$ and $|\eta|<+\infty$, there is no instability. On the other hand, when $k_{0} \rightarrow 0$ and $|\eta| \rightarrow+\infty$, there is instability (see $\S 4.4$ ).

\section{The MBF case}

If $k_{p}=\sqrt{\left(k_{1}+2 \varepsilon k_{3}\right)^{2}+k_{2}^{2}}=0$, then $k_{2}=0$ and $k_{1}=-2 \varepsilon k_{3}$. The substitution of this solution into (21) yields

$$
\hat{u}_{3}=2 \varepsilon \hat{u}_{1}, \quad \hat{b}_{3}=2 \varepsilon \hat{b}_{1} .
$$

Accordingly, the Floquet system (24) reduces to

$$
\begin{gathered}
\frac{d \hat{u}_{1}}{d \tau}=\frac{1}{\left(1+4 \varepsilon^{2}\right)} \hat{u}_{2}+\imath \eta \hat{b}_{1}, \quad \frac{d \hat{u}_{2}}{d \tau}=-\left(1-4 \varepsilon^{2}\right) \hat{u}_{1}+\imath \eta \hat{b}_{2}, \\
\frac{d \hat{b}_{1}}{d \tau}=-\hat{b}_{2}+\imath \eta \hat{u}_{1}, \quad \frac{d \hat{b}_{2}}{d \tau}=\hat{b}_{1}+\imath \eta \hat{u}_{2},
\end{gathered}
$$

with eigenvalues

$$
\begin{aligned}
& \lambda_{1,2}= \pm \imath\left[\eta^{2}+\frac{\left(1-4 \varepsilon^{2} \sqrt{1+\left(1+4 \varepsilon^{2}\right) \eta^{2}}\right)}{\left(1+4 \varepsilon^{2}\right)}\right]^{1 / 2}, \\
& \lambda_{3,4}= \pm \imath\left[\eta^{2}+\frac{\left(1+4 \varepsilon^{2} \sqrt{1+\left(1+4 \varepsilon^{2}\right) \eta^{2}}\right)}{\left(1+4 \varepsilon^{2}\right)}\right]^{1 / 2},
\end{aligned}
$$


indicating instability when $0 \leq \eta<\sqrt{4 \varepsilon^{2}-1}$. This implies that, when $\varepsilon<1 / 2$, there is no instability at $\chi= \pm 1$ with or without basic magnetic field. Note that in several geophysical or astrophysical (accretion disks) applications, the parameter of precession is small, $\varepsilon<0.2$ (see e.g., $[16])$.

When $k_{0} \equiv k_{3}=0$, the Floquet system (24) is transformed as

$$
\begin{array}{ll}
\frac{d}{d \tau}\left[k_{1} \hat{u}_{2}-k_{2}\left(\hat{u}_{1}+2 \varepsilon \hat{u}_{3}\right)\right]=0, & \frac{d \hat{u}_{3}}{d \tau}=0, \\
\frac{d}{d \tau}\left[k_{1} \hat{b}_{2}-k_{2}\left(\hat{b}_{1}-2 \varepsilon \hat{b}_{3}\right)\right]=0, & \frac{d \hat{b}_{3}}{d \tau}=0,
\end{array}
$$

indicating that, at $k_{0}=0$ and $|\eta|<+\infty$, there is no instability, as for the KBF case. However, when $k_{0} \rightarrow 0$ and $|\eta| \rightarrow+\infty$, there is instability (see $\S 4.4$ ).

\section{ASYMPTOTIC ANALYSIS}

\subsection{Change of variables}

In this subsection, we introduce new variables to facilitate subsequent calculations of the asymptotic (or perturbation) procedure for small $\varepsilon$.

In the purely hydrodynamic case, it is possible to reduce the number of dependent variables, and therefore the rank of the system of governing equations, using only solenoidal modes for the velocity disturbance. Projection of $\hat{\boldsymbol{u}}(\boldsymbol{k}, t)$ into an orthonormal frame of reference, often called Craya-Herring in the turbulence community, yields a solenoidal decomposition in terms of two-components $u^{(1)}, u^{(2)}$ (see appendix $\mathrm{V}$ here). The procedure in Fourier space, in which the solenoidal (divergence-free) property is a purely algebraic condition of orthogonality $(\boldsymbol{k} \cdot \hat{\boldsymbol{u}}=0)$, see Eqs. 21, is close to the toroidal/ poloidal decomposition in physical space (see [38] for details). In addition, the above-mentioned two components are similar to Orr-Sommerfeld-Squires variables, in terms of vertical vorticity and Laplacian of vertical velocity. This decomposition has been successfully used for RDT approach and related stability analysis in $[7,13]$, with some results (only published in French) prior to the Bayly's ones, and recalled in [7]. This decomposition applies to the magnetic disturbance field, resulting in two component $\left(b^{(1)}, b^{(2)}\right)$, as well.

Instead of working on the rank-4 system of equations for $u^{(1)}, u^{(2)}, b^{(1)}, b^{(2)}$, given in appendix $\mathrm{V}$, we prefer to generalize the similar system of four variables introduced by Lebovitz 
and Zweibel in the case of the "classical" elliptical flow case. The interest of the latter decomposition appears at vanishing basic magnetic field (i.e., $\eta=0$ ), where the induction equation reduces to

$$
d \hat{\boldsymbol{b}} / d t=\mathbf{A} \cdot \hat{\boldsymbol{b}}
$$

and therefore remains similar to the equation for the trajectories in physical space $(d \boldsymbol{x} / d \tau=$ $\mathrm{A} \cdot \boldsymbol{x})$.

The L-Z system of variables are invariant along trajectories in the limit of vanishing $\eta$. As their counterpart in the Craya-Herring system of reference, they use the Fourier components of vertical vorticity (and vertical Curl of magnetic field) and vertical velocity, but in a combined way.

The KBF case

For the KBF case, the induction equation in system (23) takes the form

$$
\frac{d \hat{b}_{1}}{d \tau}=-\hat{b}_{2}, \quad \frac{d \hat{b}_{2}}{d \tau}=\hat{b}_{1}-2 \varepsilon \hat{b}_{3}, \quad \frac{d \hat{b}_{3}}{d \tau}=0
$$

indicating that $\hat{b}_{3}$ is constant along trajectories for $\eta=0$. With the help of the equation of the wave vector (equation (15)), we easily show that the variable

$$
\left[\left(k_{1} \hat{b}_{2}-k_{2} \hat{b}_{1}\right)+2 \varepsilon k_{2} \hat{b}_{3}\right]
$$

is also constant along trajectories for $\eta=0$. When $\eta \neq 0$, the use of the two variables

$$
c_{3}=k_{1} \hat{b}_{2}-k_{2}\left(\hat{b}_{1}-2 \varepsilon \hat{b}_{3}\right), \quad c_{4}=-k_{0} \hat{b}_{3}
$$

transforms the induction equation in system (23) as follows

$$
\frac{d c_{3}}{d \tau}=\imath \eta \chi c_{1}, \quad \frac{d c_{4}}{d \tau}=\imath \eta \chi c_{2}
$$

where

$$
c_{1}=k_{1} \hat{u}_{2}-k_{2}\left(\hat{u}_{1}-2 \varepsilon \hat{u}_{3}\right), \quad c_{2}=-k_{0} \hat{u}_{3}
$$

Hence, the Floquet system (equations (23)) can be rewritten as

$$
\frac{d \boldsymbol{c}}{d \tau}=\mathbf{D} \cdot \boldsymbol{c}
$$


in the variables $\boldsymbol{c}=\left[c_{1}, c_{2}, c_{3}, c_{4}\right]^{T}$, where the non-zero components of the fourth rank matrix $\mathrm{D}$ are

$$
\begin{gathered}
D_{11}=-4 \varepsilon \frac{k_{2}\left(k_{0}-\varepsilon k_{1}\right)}{k^{2}}, \quad D_{11}+D_{22}=-4 \varepsilon \frac{k_{2} k_{3}}{k^{2}} \\
D_{12}=-2\left(1+\varepsilon \frac{k_{1}}{k_{0}}+4 \varepsilon^{3} \frac{k_{1} k_{2}^{2}}{k_{0} k^{2}}\right), \quad D_{21}=2 \frac{k_{0}\left(k_{0}-\varepsilon k_{1}\right)}{k^{2}} \\
D_{13}=D_{24}=D_{31}=D_{42}=\imath \eta \chi .
\end{gathered}
$$

The MBF case

When $\eta=0$, the induction equation in system (24) reduces to

$$
\frac{d \hat{b}_{1}}{d \tau}=-\hat{b}_{2}, \quad \frac{d \hat{b}_{2}}{d \tau}=\hat{b}_{1}, \quad \frac{d \hat{b}_{3}}{d \tau}=-2 \varepsilon \hat{b}_{2},
$$

and due to the equation for the wave vector (16), we deduce that the following two variables

$$
c_{3}=\left(k_{1}+2 \varepsilon k_{3}\right) \hat{b}_{2}-k_{2} \hat{b}_{1}, \quad c_{4}=k_{0}\left(2 \varepsilon \hat{b}_{1}-\hat{b}_{3}\right)
$$

are constants along trajectories for $\eta=0$. When $\eta \neq 0$, the use of these two variables transforms the induction equation in system (24) into the equation (29) with

$$
c_{1}=\left(k_{1}+2 \varepsilon k_{3}\right) \hat{u}_{2}-k_{2} \hat{u}_{1}, \quad c_{2}=k_{0}\left(2 \varepsilon \hat{u}_{1}-\hat{u}_{3}\right) .
$$

Accordingly, the Floquet system (24) takes the form (31) with

$$
\begin{gathered}
D_{11}=-4 \varepsilon \frac{k_{2} k_{0}}{k^{2}}-4 \varepsilon^{2} \frac{k_{1} k_{2}}{k^{2}} \frac{\left(k_{1}^{2}+k_{2}^{2}\right)}{k_{p}^{2}}+8 \varepsilon^{3} \frac{k_{2} k_{0}}{k^{2}} \frac{k_{1}^{2}}{k_{p}^{2}} \\
D_{11}+D_{22}=-4 \varepsilon \frac{k_{2} k_{0}}{k^{2}}, \quad D_{12}=-2\left(1+\varepsilon \frac{k_{1}}{k_{0}}-4 \varepsilon^{3} \frac{k_{1} k_{0}}{k^{2}} \frac{k_{2}^{2}}{k_{p}^{2}}\right), \\
D_{21}=2\left(\frac{k_{0}^{2}}{k^{2}}-\varepsilon \frac{k_{1} k_{0}}{k^{2}}+4 \varepsilon^{3} \frac{k_{1} k_{0}}{k^{2}} \frac{k_{2}^{2}}{k_{p}^{2}}\right), \\
D_{13}=D_{24}=D_{31}=D_{42}=\imath \eta \chi .
\end{gathered}
$$

As will be shown later, at sufficiently small $\varepsilon$, the matrix $\mathbf{D}$ has the same form in the both flow cases (KBF and MBF, see also [13]).

On the other hand, it should be informative for comparison to report here the non zero components of the matrix $\mathbf{D}$ in the case of the magnetoelliptical instabilities (see [35]),

$$
D_{11}=-4 \varepsilon \frac{k_{1} k_{2}}{k^{2}}, \quad D_{12}=-2, \quad D_{21}=2 \frac{k_{0}^{2}}{k^{2}}
$$




$$
D_{13}=D_{24}=D_{31}=D_{42}=\imath \eta \chi
$$

As will be shown later, for fixed value of $\eta$, the point at which occur the first harmonic instability in the KBF and MBF cases correspond to the point at which occurs the subharmonic instability in the elliptical flow case.

\subsection{The Floquet multiplier matrix}

We denote by $\boldsymbol{\Phi}(\tau)$ the fundamental matrix solution of equation (31), which is similar to the Green's function used in the purely hydrodynamic case $[6,7,13]$

$$
\frac{d}{d \tau} \boldsymbol{\Phi}+\mathbf{D} \cdot \boldsymbol{\Phi}=0, \quad \boldsymbol{\Phi}(0)=\mathbf{I}_{4}
$$

where $\mathbf{I}_{4}$ is the unit matrix, and by $\mathbf{M}=\boldsymbol{\Phi}(2 \pi)$ the Floquet multiplier matrix (see [41]). Because

$$
\frac{1}{k^{2}} \frac{d k^{2}}{d \tau}=\frac{2}{k^{2}} k_{i} \frac{d k_{i}}{d \tau}=-\frac{2}{\Omega_{0} k^{2}} k_{i} A_{j i} k_{j}=4 \varepsilon \frac{k_{2} k_{3}}{k^{2}}=-D_{i i},
$$

it found that Det $\boldsymbol{\Phi}=(K / k)^{2}$, and hence Det $\mathbf{M}=1$ (see [42]) This implies that the product of the eigenvalues of $\mathbf{M}$ is unity, say $\lambda_{1} \lambda_{2} \lambda_{3} \lambda_{4}=1$, or equivalently, $\exp \left[2 \pi\left(\sigma_{1}+\sigma_{2}+\sigma_{3}+\sigma_{4}\right)\right]=1$ since the general solution of equation (31) is a linear superposition of Floquet modes, $\boldsymbol{c}(\tau)=\mathrm{e}^{\sigma \tau} f(\tau)$ where $f(\tau)$ is periodic with period $2 \pi$ (see [41]).

If any of the eigenvalues has modulus exceeding $1(|\lambda|>1)$ there is an exponential growing for the solution of equation (31) (or (38)), and hence the system is unstable. Moreover, the Floquet system (31) possesses the property that whenever $\lambda$ is an eigenvalue of the Floquet matrix, so also are its inverse $\lambda^{-1}$ and its complex conjugate $\lambda^{*}$. The proof of this proposition is similar to the one given by Lebovitz \& Zweibel [35]. It follows that, i) in the stable case all the eigenvalues lie on the unit circle, ii) if an eigenvalue is at the onset of instability, it must have multiplicity two (or higher). Consequently, a necessary condition for the onset of linear instability is a resonance where two Floquet multipliers coincide [35]).

\section{THE SUBHARMONIC RESONANCES}

In this section, we give the main results derived from the asymptotic analysis, while the calculations yielding these results are reported in Appendices for the sake of clarity. 


\subsection{Case with circular streamlines}

When $\varepsilon=0$ (i.e., circular streamlines), the matrix $\mathbf{D}$ (say $\mathbf{D}_{0}$ ) is time-independent

$$
\mathbf{D}_{0}=\left[\begin{array}{cccc}
0 & -2 & \imath \eta \chi & 0 \\
2 \chi & 0 & 0 & \imath \eta \chi \\
\imath \eta \chi & 0 & 0 & 0 \\
0 & \imath \eta \chi & 0 & 0
\end{array}\right]
$$

with eigenvalues that are purely imaginary,

$$
\sigma_{1,2}= \pm \imath\left(1+\sqrt{1+\eta^{2}}\right) \chi, \quad \sigma_{3,4}= \pm \imath\left(1-\sqrt{1+\eta^{2}}\right) \chi
$$

Lebovitz \& Zweibel [35] noted that the first two eigenvalues correspond to "hydrodynamic modes" since they reduce, when $\eta=0$, to the eigenvalues of the purely hydrodynamic case, while the second two refer to "magnetic modes" (they are zero at $\eta=0$ ). The frequencies associated to these four eigenvalues are respectively denoted by

$$
\omega_{1,2}= \pm\left(1+\sqrt{1+\eta^{2}}\right) \chi, \quad \omega_{3,4}= \pm\left(1-\sqrt{1+\eta^{2}}\right) \chi
$$

This analysis can be supported more generally by the dispersion relation of neutral wave modes, recalled in subsection 2.1. Previous equations (41) are the dispersion laws of inertiaAlfvénic waves, given by Eq. (8), when the rotation axis is aligned with the external magnetic field, rendered nondimensional by the basic rotation.

The resonant cases for $\varepsilon=0$ are characterized by the condition $\omega_{i}-\omega_{j}=\ell$ where $\ell$ is an integer and $(i, j=1,2,3,4)$. As shown in Appendix, if $\ell \neq \pm 1$, there is no instability to leading order in $\varepsilon$, while for the magnetoelliptical instabilities, the only possibility of destabilization in the first-order analysis appears for $\ell= \pm 2$ (see $[4,35,36])$.

\subsection{Similarity between the KBF and the MBF cases}

We will now show that, at sufficiently small $\varepsilon$, there are no differences between the KBF

and MBF cases in terms of a normal mode stability analysis. Indeed, the expansion of the matrix $\mathbf{D}$ (in the Floquet system (31)) in Taylor series around $\varepsilon=0$ at fixed $\chi$, is found as

$$
D_{11}=2 \imath \varepsilon \chi \sqrt{1-\chi^{2}}\left(\mathrm{e}^{\imath \tau}-\mathrm{e}^{-\imath \tau}\right)+\mathcal{O}\left(\varepsilon^{2}\right)
$$




$$
\begin{gathered}
D_{12}=-2-\varepsilon \frac{\sqrt{1-\chi^{2}}}{\chi}\left(\mathrm{e}^{\imath \tau}+\mathrm{e}^{-\imath \tau}\right)+\mathcal{O}\left(\varepsilon^{2}\right) \\
D_{21}=2 \chi^{2}+\varepsilon \chi \sqrt{1-\chi^{2}}\left(4 \chi^{2}-1\right)\left(\mathrm{e}^{\imath \tau}+\mathrm{e}^{-\imath \tau}\right)+\mathcal{O}\left(\varepsilon^{2}\right) \\
D_{22}=\mathcal{O}\left(\varepsilon^{2}\right)
\end{gathered}
$$

for both flow cases (KBF and MBF). Therefore, if the terms of order $\mathcal{O}\left(\varepsilon^{\ell}\right)$ with $(\ell=2,3, \ldots)$ are neglected, the stability problem is the same for both the flow cases.

When considering the elliptical case and expanding the matrix $\mathbf{D}$ given by (37) in Taylor series around $\varepsilon=0$ at fixed $\chi$, we found (see also [35])

$$
\begin{aligned}
& D_{11}=\imath \varepsilon\left(1-\chi^{2}\right)\left(\mathrm{e}^{2 \imath \tau}-\mathrm{e}^{-2 \imath \tau}\right)+\mathcal{O}\left(\varepsilon^{2}\right), \quad D_{12}=-2, \\
& D_{21}=2 \chi^{2}+\varepsilon \chi^{2}\left(1-\chi^{2}\right)\left(\mathrm{e}^{2 \imath \tau}+\mathrm{e}^{-2 \imath \tau}-2\right)+\mathcal{O}\left(\varepsilon^{2}\right) .
\end{aligned}
$$

At leading order of $\varepsilon$, the time-dependent terms involving in the expansion of the matrix $\mathbf{D}$ are of the form $\exp ( \pm \imath \tau)$ for the precessing flow cases, and are of the form $\exp ( \pm 2 \imath \tau)$ for the elliptical case. Therefore, at leading order of $\varepsilon$, the instability can appear only if $\omega_{i}-\omega_{j}= \pm 1$ in the former case (see Appendix), and only if $\omega_{i}-\omega_{j}= \pm 2$ in the later case (see [35]).

\subsection{Case without magnetic field}

When $\eta=0$, an alternative formulation of the Floquet system (31) yields a Hill equation, which, at sufficiently small $\varepsilon$, reduces to a Mathieu equation (see Salhi \& Cambon [13]),

$$
\begin{gathered}
\frac{d^{2} Y}{d \tau^{2}}+\left[4 \chi^{2}-2 \varepsilon\left(3-8 \chi^{2}\right) \chi \sqrt{1-\chi^{2}} \cos \tau\right] Y=0, \\
Y=-\frac{k}{k_{p} k_{h}}\left(k_{1} \hat{u}_{2}-k_{2} \hat{u}_{1}\right), \quad k_{h}=\sqrt{k_{1}^{2}+k_{2}^{2}} .
\end{gathered}
$$

From the above Mathieu equation, one can characterize the subharmonic instability. Indeed, the stability properties of the Mathieu equation are well-known (see [39]): For small $\varepsilon$, the solutions are generally bounded, except in the vicinity of resonances defined by $\chi^{2}=n^{2} / 16$ with $n=1,2,3,4$, where the solutions are exponentially growing with growth rate of order $\varepsilon^{n}$, respectively. Because the width of the last three unstable bands is of $\mathcal{O}\left(\varepsilon^{n}\right)$ with $n \geq 2$, the Mathieu equation does not allow to determine whether instability exists near $\chi=1 / 2,3 / 4$ and $\chi=1$ for the original system (31) with $\eta=0$. Therefore, equation (44) only characterizes 
the subharmonic instability which occurs at $\chi=1 / 4$ with maximal growth rate $\sigma_{\max }$ of the form

$$
\frac{\sigma_{\max }}{\varepsilon}=\left[\left(3-8 \chi^{2}\right) \chi \sqrt{1-\chi^{2}}\right]_{n=1}=\frac{5 \sqrt{15}}{32}
$$

in agreement with the result obtained by the power statement method (Kerswell [11]). Such a result can also be recovered from the present asymptotic analysis (see $\S 4$ ).

In the presence of the magnetic field, i.e., $\eta \neq 0$, we propose to analyze the fourth order Floquet system (31) for small $\varepsilon$ by using the asymptotic (or perturbation) procedure given by Lebovitz \& Zweibel [35].

Because the interchange $\chi \rightarrow-\chi$ leads to the same set of frequencies (given by equation (40), see also $[35,36]$ ), we may consider without loss of generality that $\chi=\cos \alpha>0$. This allows us to consider only the following four resonant cases.

\subsection{Hydrodynamic modes: Case with $\omega_{1}-\omega_{2}=1$}

The substitution of the condition $\omega_{1}-\omega_{2}=1$ into the first relation in equation (41) yields $\omega_{1}=1 / 2$ or equivalently

$$
\chi=\cos \alpha=\frac{1}{2\left(1+\sqrt{1+\eta^{2}}\right)} .
$$

At vanishing basic magnetic field, i.e., $\eta=0$, equation (46) reduces to $\chi=\cos \alpha=1 / 4$ meaning that the subharmonic instability emanates from the point $P_{1}(\varepsilon=0, \chi=1 / 4)$, in agreement with previous studies $([11,13])$. When $\eta \neq 0$, the point $P_{1}$ that lies to the $\varepsilon=0$ axis approaches the point $P(0,0)$ since the value of $\chi$ yielded by $(46)$ decreases with increasing $\eta$, and approaches zero (so that $k_{0} \rightarrow 0$ ) for $\eta \rightarrow+\infty$ (see figure 2-a). Recall that, when $|\eta|<+\infty$ and $\chi=\cos \alpha=0$, the Floquet system (31) is stable (see equations (25) and (26)).

Regarding the maximal growth rate $\sigma_{\max }$ (defined by equation (79) in Appendix IV) of the "hydrodynamic" subharmonic instability, it takes the form (see equation (80)),

$$
\frac{\sigma_{\max }}{\varepsilon}=\left|1-\chi-2 \chi^{2}\right| \frac{\sqrt{1-\chi^{2}}}{4 \chi \sqrt{1+\eta^{2}}}
$$

In the limit $\eta \rightarrow 0$ (the pure hydrodynamic limit), one has $\chi=1 / 4$ as already noted, and hence, $\sigma_{\max } / \varepsilon=5 \sqrt{15} / 32$, in agreement with (45). Note that, at $\chi=1 / 4$, equations $(47)$ and ((45) yields the same value. As it can be expected, $\sigma_{\max } / \varepsilon$ decreases as $\eta$ increases 
approaching $\varepsilon / 2$ when $\eta \rightarrow \infty$ (see figure 2-b). This means that the basic magnetic field acts to reduce the maximal growth rate of the "hydrodynamic" subharmonic instability.

As for the width $\delta=\left(\nu_{+}-\nu_{+}\right) \varepsilon$ of the "hydrodynamic" subharmonic instability, it is of the form (see equation (81)

$$
\delta=\left|1-\chi-2 \chi^{2}\right| \frac{\sqrt{1-\chi^{2}}}{\sqrt{1+\eta^{2}}} \varepsilon
$$

with $\nu_{+}=-\nu_{-}=\delta /(2 \varepsilon)$ (the slopes $\nu_{ \pm}$are defined by equation (78) in Appendix IV). The above relation shows that, at fixed $\varepsilon$, the width $\delta$ decreases as $\eta$ increases: It varies between $\delta=(5 \sqrt{15} / 32) \varepsilon$ at $\eta=0$ and $\delta \rightarrow 0$ as $\eta \rightarrow \infty$ (see figure 2-c). In the $(\varepsilon, \chi)$ plane, the instability band is symmetrical with respect to the axis $\varepsilon=1 /\left[2\left(1+\sqrt{1+\eta^{2}}\right)\right]$. Note that in the case of the elliptical instability, it has been shown that the subharmonic "hydrodynamic" instability band is not symmetrical with respect to the axis $\varepsilon=1 /\left[1+\sqrt{1+\eta^{2}}\right]$ because $\left|\nu_{-}\right|<\left|\nu_{+}\right|$. Its maximal growth rate varies between $(9 / 16) \varepsilon$ in the pure-hydrodynamic limit $\eta=0($ see $[4,34,35])$ and approaches $\varepsilon / 4$ when $\eta \rightarrow \infty$ (see figure 3 and [35]).

\subsection{Mixed modes: Case with $\omega_{1}-\omega_{3}=1$}

The substitution of (41) into the relation $\omega_{1}-\omega_{3}=1$ yields $\omega_{1}=\chi+1 / 2$ and $\omega_{3}=\chi-1 / 2$, and hence,

$$
\chi=\cos \alpha=\frac{1}{2 \sqrt{1+\eta^{2}}} .
$$

In the $(\varepsilon, \chi)$ plane, the point $P_{2}\left(\varepsilon=0, \chi=1 /\left(2 \sqrt{1+\eta^{2}}\right)\right)$, from which this instability emanates, approaches the point $P(0,0)$ as $\eta \rightarrow \infty$ (see figure $2-\mathrm{b}$ ). This subharmonic instability represents a "mixed" mode since, at $\eta=0, \omega_{3}$ becomes zero while $\omega_{1}$ reduces to unity. We note that, at $\eta=0$, the instability emanating from $P_{2}(0, \chi=1 / 2)$ is rather harmonic since it is of order $\varepsilon^{2}$ (see [13]). The maximal growth rate of the "mixed" subharmonic instability takes the form (see equation (82) in Appendix IV),

$$
\frac{\sigma_{\max }}{\varepsilon}=\frac{\left|1-4 \chi^{2}\right| \sqrt{1-\chi^{2}}}{4 \sqrt{1+\eta^{2}}}=\frac{\eta^{2} \sqrt{3+4 \eta^{2}}}{8\left(1+\eta^{2}\right)^{2}} .
$$

For fixed $\varepsilon$, the maximal growth rate is zero at $\eta=0$, and increases for $0 \leq \eta<\eta_{m}=$ $(\sqrt{3+\sqrt{33}}) / 2$, reaches to the maximal value

$$
\frac{(3+\sqrt{33}) \sqrt{6+\sqrt{33}}}{2(7+\sqrt{33})^{2}}
$$


at $\eta=\eta_{m}$ and decreases for $\eta_{m}<\eta$ approaching zero as $\eta \rightarrow \infty$ (see figure 2-b). The width of the "mixed" subharmonic instability is found as (see equation (83) in Appendix IV)

$$
\delta=\left(\nu_{+}-\nu_{-}\right) \varepsilon=\frac{\chi\left|1-4 \chi^{2}\right| \sqrt{1-\chi^{2}}}{\sqrt{1+\eta^{2}}} \varepsilon=\frac{\eta^{2} \sqrt{3+4 \eta^{2}}}{4\left(1+\eta^{2}\right)^{5 / 2}} \varepsilon,
$$

with $\nu_{+}=-\nu_{+}=\nu /(2 \varepsilon)$. This implies that, in the $(\varepsilon, \chi)$ plane, the "mixed" subharmonic instability is symmetrical with respect to the axis $\varepsilon=1 /\left[2 \sqrt{1+\eta^{2}}\right]$. The bandwidth has a maximum at $\eta(\sqrt{3+\sqrt{201}}) / 4$ and approaches zero as $\eta \rightarrow \infty$ (see figure 2-c).

For the elliptical case studied by Lebovitz \& Zweibel [35]), the "mixed" instability emanates from the point $P_{2}\left(0, \chi=1 / \sqrt{1+\eta^{2}}\right)$ (see figure 3 -a) with a maximal growth rate that increases when $\eta$ increases: It takes a zero value at $\eta=0$ and approaches $\varepsilon / 4$ as $\eta \rightarrow \infty$ (see figure 3-b and [35]).

\subsection{Magnetic modes: Case with $\omega_{4}-\omega_{3}=1$}

The substitution of the expression of $\omega_{3}$ and $\omega_{4}$ given by equation (41) into the relation $\omega_{4}-\omega_{3}=1$ yields

$$
\chi=\cos \alpha=\frac{1}{2\left(\sqrt{1+\eta^{2}}-1\right)} .
$$

Because $\chi \leq 1$, the resonance can occur only when

$$
\eta>\frac{\sqrt{5}}{2}
$$

This subharmonic instability is due to the resonance between the magnetic modes and it is called the subharmonic "magnetic" instability. In the $(\varepsilon, \chi)$ plane, the point $P_{3}(\varepsilon=0, \chi=$ $\left.1 / 2\left(\sqrt{1+\eta^{2}}-1\right)\right)$ at which the "magnetic" instability occurs, also approaches the point $P(0,0)$ as $\eta \rightarrow \infty$ (see figure $2-\mathrm{a}$ ). The fact that $\eta>\sqrt{5} / 2$ implies that the "magnetic" stability cannot emanate exactly from the point $(\chi=1, \varepsilon=0)$. Recall that, at $\chi=1$ so that $k_{p}=0$, there is no instability (see $\S 2.3$ ).

When $\sqrt{5} / 2>\eta$, the maximal growth rate of the subharmonic "magnetic" instability is of the form (see equation (84) in Appendix IV)

$$
\frac{\sigma_{\max }}{\varepsilon}=\frac{\left|1+\chi-2 \chi^{2}\right| \sqrt{1-\chi^{2}}}{4 \chi \sqrt{1+\eta^{2}}} .
$$



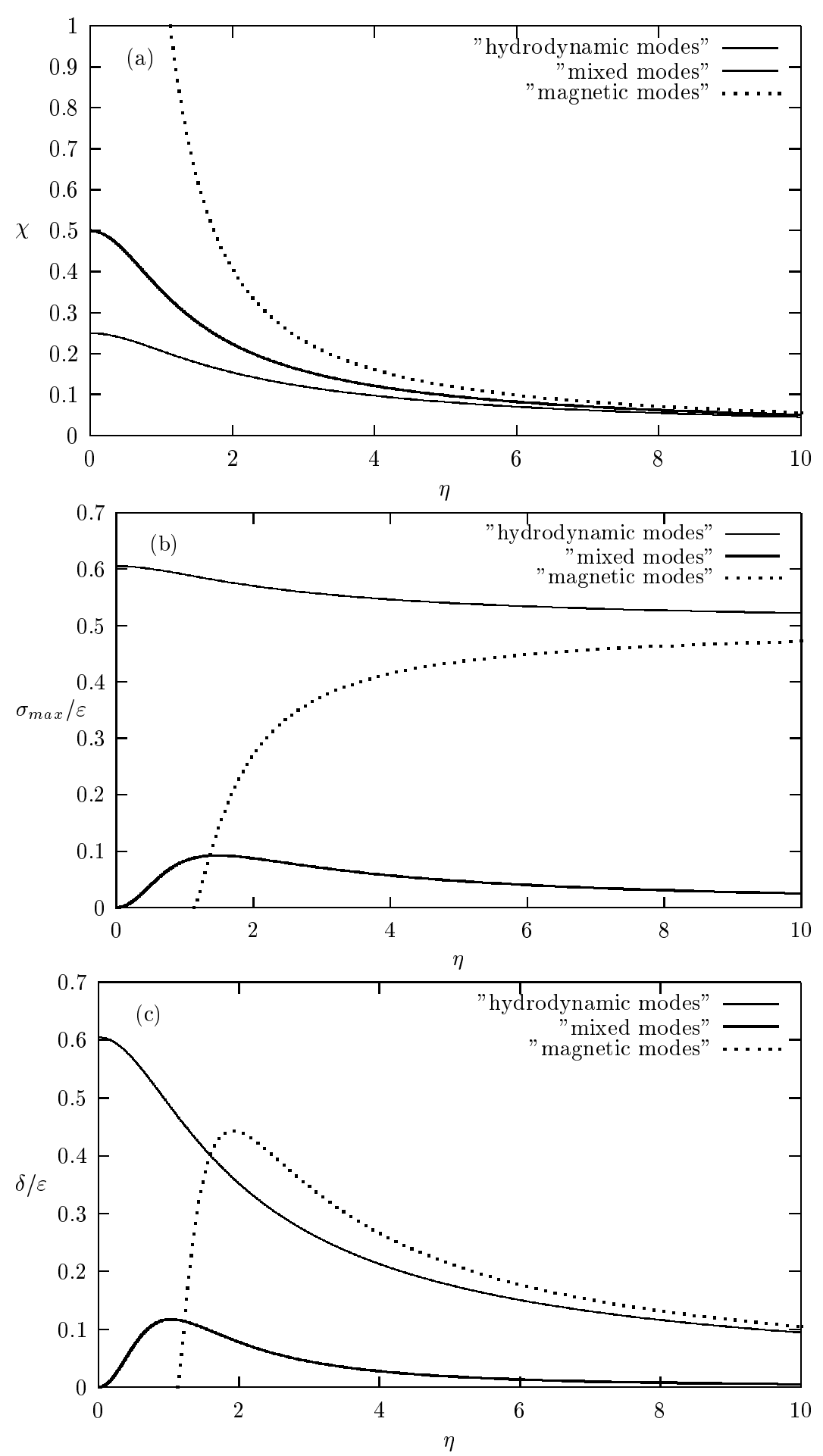

FIG. 2: (a) Dependence of the points where the three subharmonic instabilities occur on the magnetic parameter $\eta$ (equations (46), (49), (52)); (b) Dependence of the maximal growth rate $\sigma_{\max }$ normalized by the precession parameter $\varepsilon$ of the three subharmonic instabilities on the magnetic parameter $\eta$ (equations (47), (50), (54)); (c) Dependence of the width of the three subharmonic instabilities normalized by the precession parameter $\varepsilon$ on the magnetic parameter $\eta$ (equations (48), (51), (55)). 
Therefore, for fixed $\varepsilon$, the maximal growth rate increases as $\eta$ increases, approaching $\varepsilon / 2$ as $\eta \rightarrow \infty$ (see figure 2-b). The expression of the width of the "magnetic" instability is found as (see equation (85) in Appendix IV)

$$
\delta=\frac{\left|1+\chi-2 \chi^{2}\right| \sqrt{1-\chi^{2}}}{\sqrt{1+\eta^{2}}} \varepsilon,
$$

with $\nu_{+}=-\nu_{-}=\delta /(2 \varepsilon)$ signifying that, in the $(\varepsilon, \chi)$ plane, the subharmonic magnetic instability band is symmetrical with respect to the axis $\varepsilon=1 / 2\left(\sqrt{1+\eta^{2}}-1\right)$. For the sake of clarity, we do not report here the expression of $\delta$ in function of $\eta$, but we indicate that $\delta$ has a maximum value at $\eta \approx 1.9$ and approaches zero when $\eta \rightarrow \infty$ (see figure 2 -c).

For the elliptical case, the subharmonic "magnetic" instability appears only when $\eta>$

$\sqrt{3}$ : It emanates from the point $P_{3}\left(\varepsilon=0, \chi=1 /\left(\sqrt{1+\eta^{2}}-1\right]\right)$ with maximal growth rate approaching $\varepsilon / 4$ as $\eta \rightarrow \infty$ (see figure 3 and [35]).

Finally, considering the case with $\omega_{1}-\omega_{4}=1$. The use of (41) leads to $\chi=\cos \alpha=1 / 2$, but, at first order in $\varepsilon$, there is no instability associated with this resonance (see the end of Appendix IV).

\section{DISCUSSION}

In this section, the asymptotic analysis results are discussed and their validity when $\varepsilon(\leq 0.25)$ is not small. Recall that the domain $0<\varepsilon \leq 0.25$ covering most of geophysical and astrophysical applications (see e.g. [16]) is addressed in connection with the numerical results. For fixed values of the parameters $\varepsilon$ and $\eta$ and $0<\chi<1$, the Floquet system (31) is solved numerically over one period $(0 \leq \tau \leq 2 \pi)$ (using the fourth order Runge-Kutta method) to compute $\mathbf{M}(2 \pi)$ and to determine its eigenvalues (using the QZ method) $\Lambda_{i}$ $(i=1,2,3,4)$, and hence, the growth rate $\sigma_{i}$,

$$
\sigma_{i}=\frac{1}{2 \pi} \log \Lambda_{i} \quad(i=1,2,3,4) .
$$

Recall that if any of the eigenvalues satisfies $\left|\Lambda_{i}\right|>1$ the system is unstable.

\subsection{The validity of the asymptotic analysis when $\varepsilon$ is not small}

Figure 4 shows the subharmonic instability bands in the $(\varepsilon, \chi)$ plane for $\eta=0,1,2$ and $\eta=5$, respectively. The choice of these values is justified along the following analysis. At 

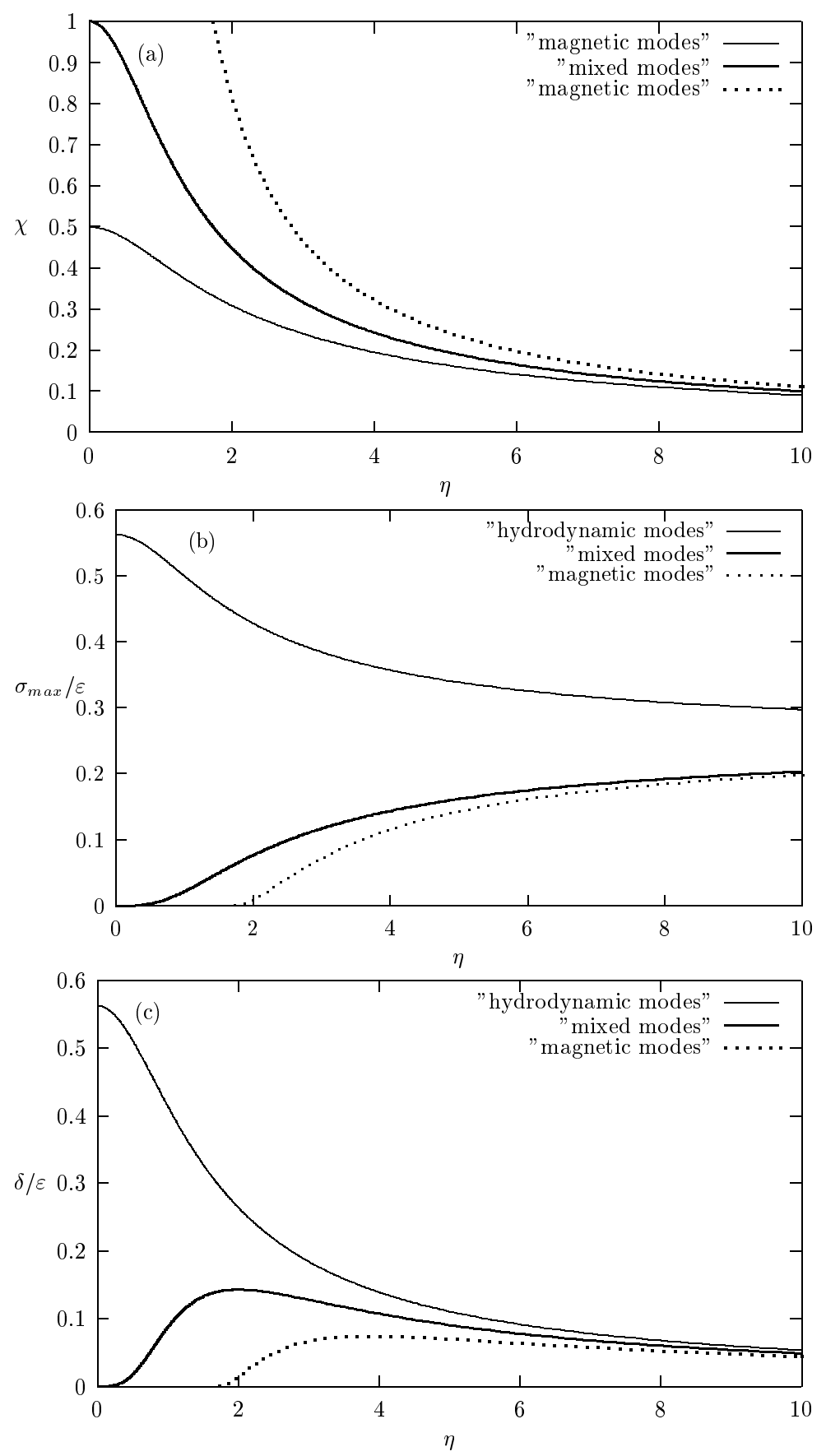

FIG. 3: (a) Dependence of the points where the first subharmonic magnetoelliptic instabilities occur on the magnetic parameter $\eta$ (see Lebovitz \& Zweibel [35]), "hydrodynamic modes": $\chi=$ $\left[1+\sqrt{1+\eta^{2}}\right]^{-1}$, "mixed modes": $\chi=\left[\sqrt{1+\eta^{2}}\right]^{-1}$, "magnetic modes": $\chi=\left[\sqrt{1+\eta^{2}}-1\right]^{-1} ;(\mathrm{b})$ Variation of $\sigma_{\max } / \varepsilon$ versus $\eta$ (see [35]), "hydrodynamic modes": $\sigma_{\max } / \varepsilon=(1+\chi)^{2} / 4$, "mixed modes": $\sigma_{\max } / \varepsilon=(1-\chi)^{2} / 4$, "magnetic modes": $\sigma_{\max } / \varepsilon=(1-\chi)^{2} / 4$; (c) Variation of $\delta / \varepsilon$ versus $\eta$ (see [35]). "hydrodynamic modes": $\delta / \varepsilon=\chi(1+\chi)^{2} / 2$, "mixed modes": $\delta / \varepsilon=\chi\left(1-\chi^{2}\right)^{2} / 2$, "magnetic modes": $\delta / \varepsilon=\chi(1-\chi)^{2} / 2$. 
vanishing magnetic field $(\eta=0)$, only the "hydrodynamic" modes exist and the subharmonic ones emanates from the point

$$
\chi=\frac{1}{\left(1+2 \sqrt{1+\eta^{2}}\right)}=0.25
$$

of the $\varepsilon=0$ axis. At $\eta=1$, there are only "hydrodynamic" and "mixed" instabilities. The subharmonic modes occur at the points

$$
\chi \approx 0.207, \quad \chi=\frac{1}{2 \sqrt{1+\eta^{2}}} \approx 0.356,
$$

respectively since the "magnetic" instability appears only for $\eta>\sqrt{5} / 2$. In addition, the choice $\eta=1$ can be justified by the fact that the width of the "mixed" instability band approaches the maximal value which occurs at $\eta \approx 1.036$ (see $\S 4.4$ ). The width of the "magnetic" instability band. The choice $\eta=2>\sqrt{5} / 2$ is due to the fact that the width of the subharmonic "magnetic" mode is maximal at $\eta \approx 1.9$ (see section 4.5 ). At $\eta=2$, the three subharmonic instabilities ("hydrodynamic", "mixed" and "magnetic") are present and they emanate from the points

$$
\chi \approx 0.155, \quad \chi \approx 0.224, \quad \chi=\frac{1}{2\left(\sqrt{1+\eta^{2}}-1\right)} \approx 0.405
$$

of the $\varepsilon=0$ axis, respectively. As for the value $\eta=5$, it has been chosen to illustrate the fact that the "hydrodynamic" and the "magnetic" subharmonic instabilities can merge for $\varepsilon<0.25$. Indeed, at sufficiently $\varepsilon$, we assume that the variation of $\chi$ versus $\varepsilon$ is linear (see 61 in Appendix I and [35]), $\chi(\varepsilon)=\chi(0)+\nu_{ \pm} \varepsilon$. Therefore, the use of (48) and (55) allows us to deduce that the "hydrodynamic" and the "magnetic" instabilities merge when

$$
\varepsilon \geq\left[\eta^{2}\left(\nu_{+h}-\nu_{-m}\right)\right]^{-1}
$$

in which $\nu_{+h}=\delta /(2 \varepsilon)$ ( $\delta$ given by equation (48)) and $\nu_{-m}=-\delta /(2 \varepsilon)$ (given by equation (55)) denote the positive and negative slopes corresponding to the "hydrodynamic" and the "magnetic" instability bands, respectively (see also figure 4-d). At $\eta=5$, the "hydrodynamic", "mixed" and "magnetic" subharmonic resonances occur at

$$
\chi \approx 0.082, \quad \chi \approx 0.098, \quad \chi \approx 0.122,
$$

respectively, of the $\varepsilon=0$ axis. It is clear from figure 4 that, for $\varepsilon \leq 0.25$, the stability bound- 

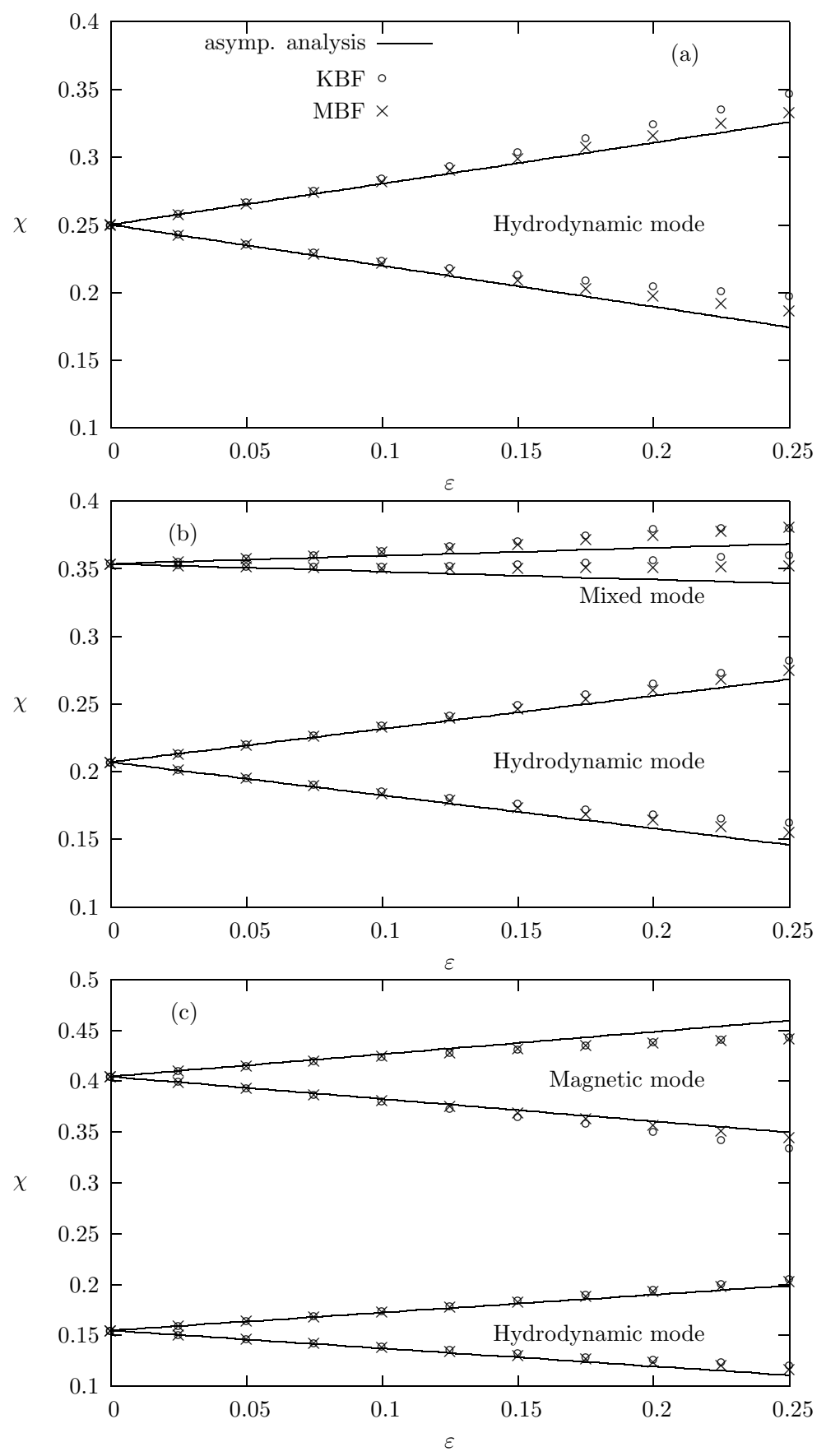

FIG. 4: Stability boundaries in the $(\varepsilon, \chi)$ plane for the KBF and MBF cases. Comparison of the asymptotic analysis results with the numerical ones for (a) $\eta=0$ (purely hydrodynamic mode); (b) $\eta=1$ (only the hydrodynamic and mixed modes are present); (c) $\eta=2$ (the three subharmonic instabilities are present but only the boundaries of the hydrodynamic and magnetic modes are presented since the width of the mixed mode is relatively small); (d) $\eta=5$ (The hydrodynamic and magnetic instabilities merge for $\varepsilon \geq 0.2$ ). 
aries are well reproduced by the analytical results except those for the "mixed" instability band at $\varepsilon>0.1$. In that case, the agreement is not quite good as for the "hydrodynamic" and the mixed ones. Note that the width of the "mixed" instability is relatively thin (see figure 2-c). To more illustrate the agreement between the analytical results and the numerical ones for $\varepsilon<0.25$, figure 5 -a compares the variation of $\delta / \varepsilon$ versus $\eta$ for the subharmonic "hydrodynamic" instability (see equation (48)) with the numerical results obtained for several values of $0 \leq \varepsilon \leq 0.25$. Figure 5 -b shows the variation of $\sigma_{\max } / \varepsilon$ versus $\eta$ for the subharmonic "hydrodynamic" instability which is the most unstable one (see equation (47) and figure 2 -c). We have also reported in this figure the numerical results at $\varepsilon=0.2$ for both the flow cases (KBF and MBF). As it can be seen, equation (47) well approximates the maximal growth rate of the "hydrodynamic" instability even if $\varepsilon$ is not small. Recall that, at $\eta \gg 1$, the maximal growth rate of the "hydrodynamic" and "magnetic" instabilities approach $\varepsilon / 2$ (see figure 2-b).

\subsection{Harmonic resonances}

While, at sufficiently small $\varepsilon$, one obtains the same results considering either the KBF case or the MBF case, at order $\varepsilon^{\ell}$ with $\ell=2,3, \ldots$, there are differences between these two flow cases as illustrated by figures 6 and 7 displaying $(\varepsilon+\Re \sigma)$ versus $\chi=\cos \alpha$ for fixed $\varepsilon$ $(0 \leq \varepsilon \leq 0.4)$, and $\eta=1$ (figure 6) and $\eta=5$ (figure 7). As indicated in section 4.1, the resonant cases for $\varepsilon=0$ are characterized by the condition

$$
\omega_{i}-\omega_{j}=\ell, \quad(i, j=1,2,3,4)
$$

where $\ell$ is an integer. According to the asymptotic analysis, at leading order of $\varepsilon$, the modes for which

$$
\omega_{1}-\omega_{2}=1, \quad \omega_{1}-\omega_{3}=1, \quad \omega_{3}-\omega_{4}=1,
$$

are exited by the precession and due to this mechanism, subharmonic instabilities appear, in agreement with the numerical results which also show the presence of harmonic resonances when

$$
\begin{gathered}
\omega_{1}-\omega_{2}=\ell_{1} \in \mathcal{E}_{1}=\left\{2, \ldots,\left\lfloor 2\left(\sqrt{1+\eta^{2}}+1\right)\right\rfloor\right\}, \\
\omega_{1}-\omega_{3}=\ell_{2} \in \mathcal{E}_{2}=\left\{2, \ldots,\left\lfloor 2 \sqrt{1+\eta^{2}}\right\rfloor\right\}
\end{gathered}
$$



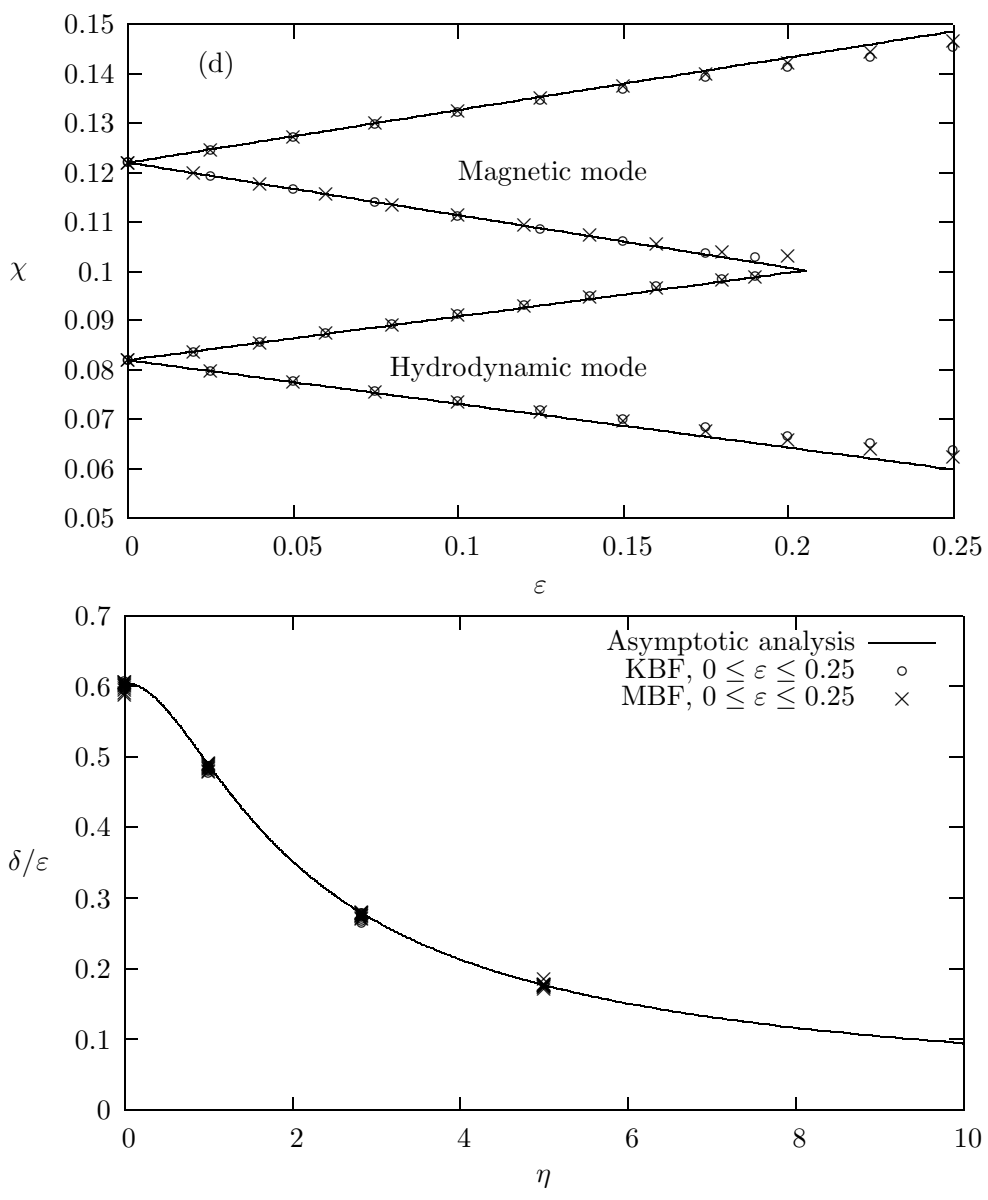

(a)

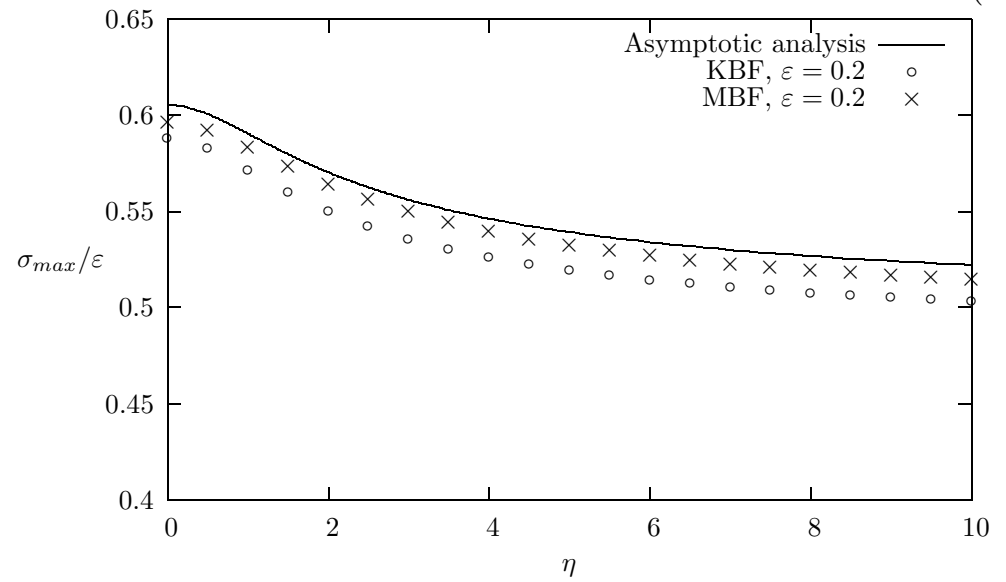

(b)

FIG. 5: The figure compares the asymptotic analysis results with the numerical ones $(\varepsilon \leq 0.25$. $)$ for the subharmonic "hydrodynamic" instability (which is the most unstable one) in both flow cases (KBF and MBF). (a): Variation of $\delta / \varepsilon$ versus $\eta$. (b) variation of $\sigma_{\max } / \varepsilon$ versus $\eta$. 


$$
\omega_{3}-\omega_{4}=\ell_{3} \in \mathcal{E}_{3}=\left\{2, \ldots,\left\lfloor 2\left(\sqrt{1+\eta^{2}}-1\right)\right\rfloor\right\} .
$$

Here $\lfloor\cdot\rfloor$ is the floor function. For instance, at $\eta=1<\sqrt{5} / 2$, only the "hydrodynamic" and "mixed" resonances are present, and according to relation (57), there are three harmonic "hydrodynamic" resonances $\left(\ell_{1} \in\{2,3,4\}\right)$ occurring at

$$
\chi=\frac{\ell_{1}}{2\left(\sqrt{1+\eta^{2}}+1\right)} \approx 0.414,0.621,0.828,
$$

respectively, whereas, there is only one harmonic "mixed" resonance $\left(\ell_{2} \in\{2\}\right)$ occurring at

$$
\chi=\frac{\ell_{2}}{2 \sqrt{1+\eta^{2}}} \approx 0.707
$$

(see figure 6-a,b,d). The third harmonic "hydrodynamic" resonance is of order $\mathcal{O}\left(\varepsilon^{4}\right)$ and hence it is very thin (see figure 6-c). The comparison made for several values of $\eta$ shows that the "hydrodynamic" and "magnetic" harmonic instability bands are larger in the MBF case than in the KBF and inversely when considering the "mixed" harmonic instability bands as illustrated by figure 7 displaying $\varepsilon+\Re \sigma$ versus $\chi$ for fixed $0 \leq \varepsilon \leq 0.4$ and $\eta=5$. In that case and according to relation (57), the first harmonic "hydrodynamic", "mixed" and "magnetic" modes occur at $\chi \approx 0.164,0.196,0.244$, respectively, while the second ones occur at $\chi \approx 0.246,0.294,0.366$, respectively.

For the elliptical case, the integers $\ell_{1}, \ell_{2}$ and $\ell_{3}$ given by (57) must be even, and hence at $\eta=1$, there are two subharmonic "hydrodynamic" and one "mixed" modes occurring at $\chi \approx 0.414,0.828$ and $\chi \approx 0.707$, respectively (see figure $6-\mathrm{c}$ ). The subharmonic instability bands in the elliptical case are larger than their counterparts (those associated to the even integers in $\mathcal{E}_{1}, \mathcal{E}_{2}$ and $\mathcal{E}_{3}$ ) in the $\mathrm{KBF}$ and $\mathrm{MBF}$ (see also figure 7 ). Finally, we note that the maximal growth rate for the precessing flow cases is more important than the one for the flow with elliptical streamlines (see figures 2 and 3 ).

\subsection{Upper bound for the magnetic field strength}

Lebovitz and Zweibel [35] have applied the results of the magnetoelliptic instability analysis to a system of finite vertical thickness $H$ (such as the accretion disks). They showed that, in that case, the instability would operate when the Alfvén speed $V_{A}=B_{0} / \sqrt{\rho \mu_{0}}$ does not exceed the critical value

$$
V_{A} \leq \sqrt{3} \frac{\Omega_{0} H}{C}
$$



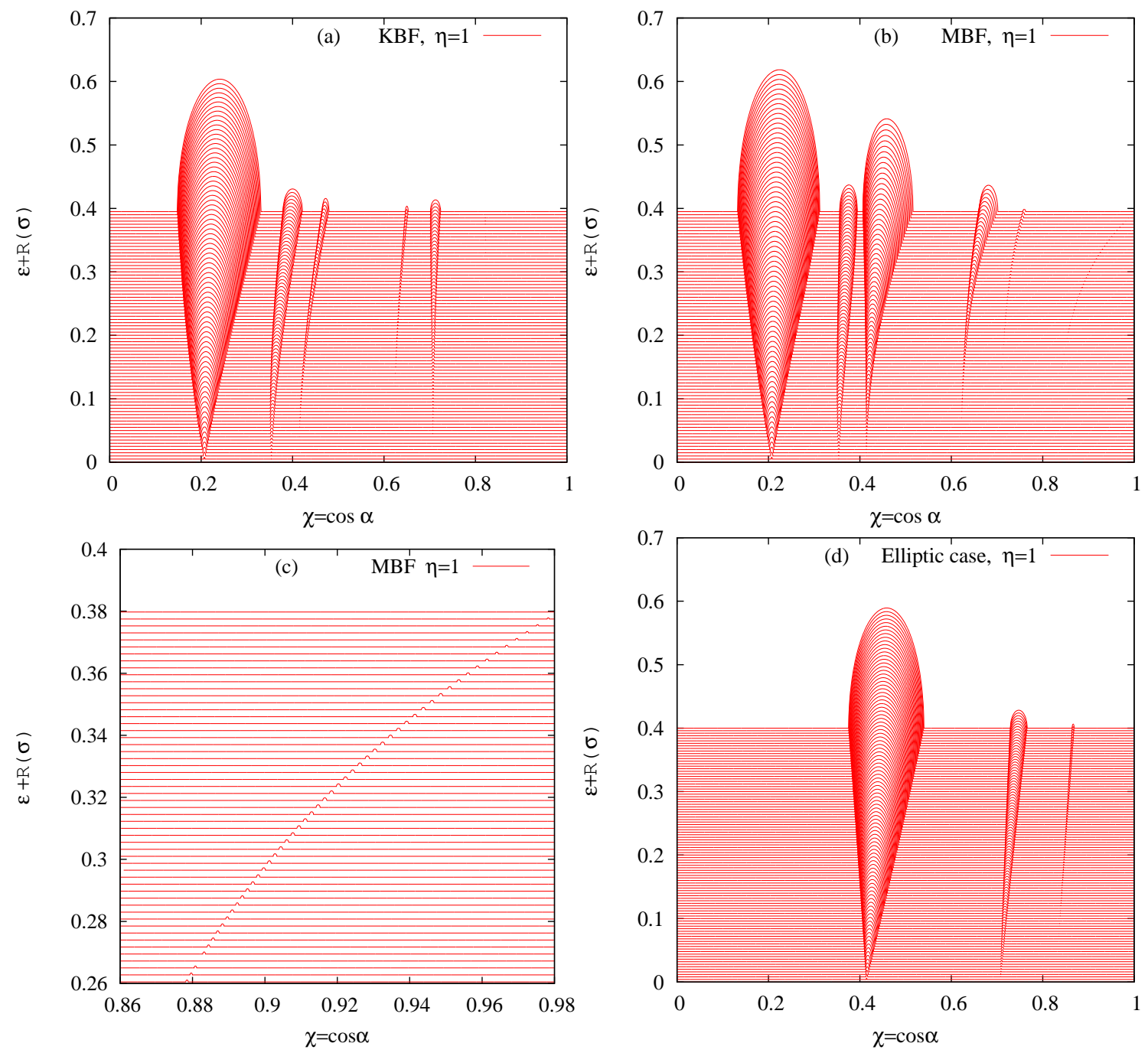

FIG. 6: Variation of $\varepsilon+\Re(\sigma)$ versus $\chi=\cos \alpha$ for fixed $0 \leq \varepsilon \leq 0.4$ and $\eta=1$. (a) the KBF case, (b)-(c) the MBF case, (d) the case with elliptical streamlines.

where $C$ is a factor of order unity. More recently, Mizerski \& Bajer [36], who studied the effects of the Coriolis force on the magnetoelliptic instability, have showed that rotation significantly modifies the above condition: The bound on $B_{0}$ is eased for cyclonic rotation (see their equation (4.22)).

We note that for the elliptical case, both the vertical space coordinate $x_{3}$ and the vertical wavenumber $k_{3}$ are time independent. Therefore, the product $k_{3} x_{3}$ is also time-independent. In contrast, for both the precessing shear cases considered in the present study, only one of these two variables is time independent. For instance, in the MBF case, $k_{0}=k_{3}=K_{3}$ is 


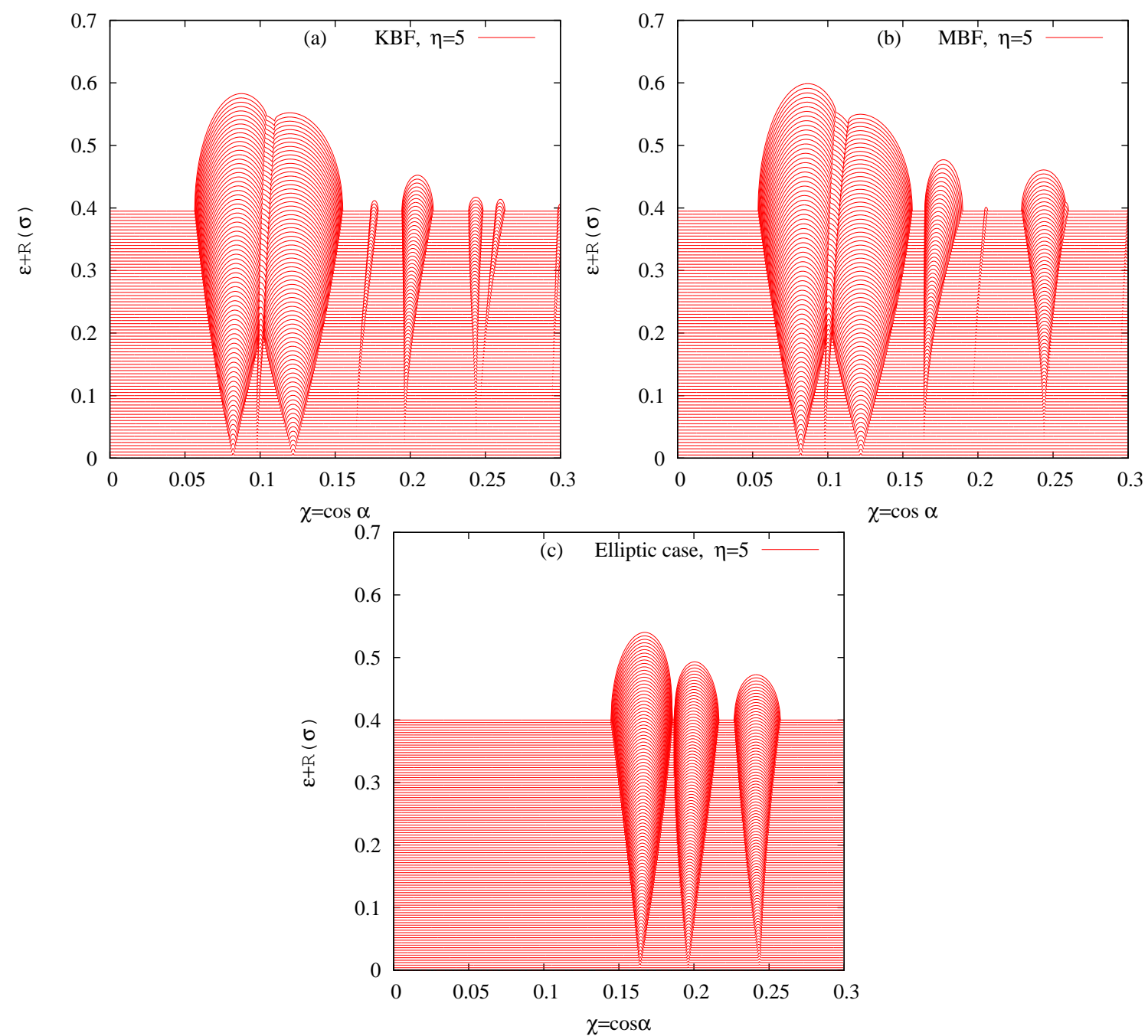

FIG. 7: Variation of $\varepsilon+\Re(\sigma)$ versus $\chi=\cos \alpha$ for fixed $0 \leq \varepsilon \leq 0.4$ and $\eta=5$. (a) the KBF case, (b) the MBF case, (d) the case with elliptical streamlines.

time-independent, while

$$
x_{3}=X_{3}+2 \varepsilon\left(x_{1}-X_{1}\right),
$$

is time-dependent since the trajectory equation, $d \boldsymbol{x} / d t=\mathbf{A} \cdot \boldsymbol{x}$, yields

$$
x_{1}=x_{p} \cos \tau^{\prime}, \quad x_{2}=x_{p} \sin \tau^{\prime}, \quad \tau^{\prime}=\Omega_{0} t+\arctan \left(X_{2} / X_{1}\right)
$$

where $\boldsymbol{X}$ is the position vector at $t=0$ and $x_{p}=\sqrt{X_{1}^{2}+X_{2}^{2}}$. Therefore, one may consider a precessing $2-\mathrm{D}$ system $\left(X_{1} \rightarrow 0\right)$ with finite vertical thickness $H$,

$$
\left|x_{0}\right|=\left|x_{3}-2 \varepsilon x_{1}\right|=\left|X_{3}\right| \sim H .
$$


Therefore, the vertical wavenumber $k_{0}=k_{3}$ cannot be smaller than $C / H$ in order that subharmonic instability operates,

$$
v_{A} \leq \frac{\sqrt{5}}{2} \frac{\Omega_{0} H}{C}
$$

Indeed, both the "hydrodynamic" and "mixed" instabilities require $\chi \eta \leq 1 / 2$ (see equations (48) and 51), while the "magnetic" instability requires (see equation (55)),

$$
\chi \eta=\frac{k_{0} B_{0}}{\Omega_{0} \sqrt{\rho \mu_{0}}}=\frac{k_{0} v_{A}}{\Omega_{0}} \leq \frac{\sqrt{5}}{2} .
$$

In the KBF case, $x_{0}=x_{3}=X_{3}$ is time-independent, whereas, $k_{3}=k_{0}-2 \varepsilon k_{1}$ is timedependent (see equation (15)). Therefore, if one considers a system of finite vertical thickness $H$ and initial perturbations with $K_{1}=0$, then the wavenumber $k_{0} /\left(=k_{3}+2 \varepsilon k_{1}=K_{3}\right)$ cannot be smaller than $C / H$, and hence, one obtains the condition (59). The critical value for the magnetic field strength in the precessing sheared cases (given by equation (60)) is not very different to the critical value in the magnetoelliptical instabilities (see equation (58)), both are comparable to the maximum value of the field at which the magnetorotationnal instability (MRI) can operate (see [22, 35]).

\section{CONCLUDING REMARKS}

Extension of the study of precessing flows with external magnetic field and coupled induction equation for the magnetic field has been performed here in a linear stage. Analogies and differences of "magneto-precessional" instabilities with magneto-elliptical ones can thus be discussed, at least in the unbounded flow case. Even in the purely hydrodynamic case, elliptical streamlines are found in both cases, but the occurrence of ellipticity in the precessional case merits to be recalled. As shown by [13], the gyroscopic torque induced by the misalignment of the precession axis with the main solid body rotation axis can be exactly balanced by an additional plane shear, and this base shear, of the same magnitude as the Poincaré parameter $\varepsilon$, combined with the basic rotation, yields elliptical streamlines. The

previous "hydro" analysis allowed us to identify two cases of balancing shear, yielding the KBF case with horizontal plane shear and the MBF case with vertical one. Additional shear is in agreement with previous physical experiments $([16,37])$. For the MBF case, the basic absolute vorticity aligns with the vertical axis, whereas, for the KBF case, it has an horizontal component (along $x_{1}$ ) of the form $4 \varepsilon \Omega_{0}$, in addition to the vertical component 
$\left(2 \Omega_{0}\right)$. Extension to the magnetic case of these exactly balanced contributions of shear, for the $B_{0}=0$ case, to absolute vorticity, related to Craik's "admissibility conditions" as well, have nontrivial consequences: The external magnetic field must align with the basic absolute vorticity. Accordingly, for the KBF case, the basic magnetic field must have an horizontal component (along $x_{1}$ ) of the form $2 \varepsilon B_{0}$ in addition to the vertical component $B_{0}$. The horizontal component can be seen as generated by the vertical magnetic field and the horizontal shear.

For more classical elliptical flow instabilities, and their recent extension to magnetoelliptical ones, the ellipticity of basic streamlines is given a priori, with for instance the parameter $E$ given in Eq. (13), and only horizontal ellipses are considered, whereas precession can cause both ellipticity and departure of the plane of trajectories from horizontal. Even if the small parameter $\varepsilon$ can be defined both in the precessional case and in the "classical elliptical" case, its physical origin is very different.

Linear stability has been investigated for disturbances to the base flow in terms of baseflow-advected Fourier modes. In the presence of closed streamlines for the base-flow, ellipses in all cases here, this method yields time-periodicity for the wave vector, so that the analysis amounts to solve a linear system of equations with time-periodic coefficients, and a Floquet problem is called into play. This Floquet system of equations has been written in a suitable frame obtained by a systematic change of variables guided by symmetry and the existence of invariants of motion. At sufficiently small precessing parameter $\varepsilon$, the Floquet system, which is the same for the two precessing flow cases (KBF and $\mathrm{MBF}$ ), has been analyzed by using an asymptotic method first introduced in the study of the magnetoelliptical instabilities by Lebovitz and Zweibel [35]. We have shown that, in the $(\varepsilon, \chi)$ plane, the point where the first harmonic resonances occur for the precessing shear flow cases correspond to the point where the subharmonic resonances occur for the elliptical flow case. Definition of three types of modes, "hydro", "magnetic" and "mixed" (e.g. [35]) follows from the marginal stability analysis at vanishing ellipticity or precession (or $\varepsilon$ parameter) and small $\eta$. We have computed the thresholds, maximum instability growth rates and bandwidths for the different involved modes mainly as function of the magnetic parameter $\eta$. We have shown that the magnetic field acts on the "hydrodynamic" subharmonic instability that is induced by the precession in the absence of the magnetic field ([11-13]), by reducing its width and its growth rate, while other (mixed and magnetic) subharmonic modes appear. The subharmonic 
magnetic mode occurs only when the magnetic parameter $\eta$ is greater than $\sqrt{5} / 2$ and at large $\eta(\gg 1)$ the maximal growth rate of both the "hydrodynamic" and "magnetic" modes approaches $\varepsilon / 2$, while the one of the subharmonic "mixed" mode approaches zero.

Fig. 2, for the magneto-precessional, MBF or KBF case, and Fig. 3, for the magnetoelliptical case, shows significant differences in terms of location of subharmonic instability, maximum growth rate and bandwidth. As the most important difference, the growth rate of the magnetic mode largely exceeds the one of the mixed mode at large $\eta$ in our "precessional case", and becomes close to the growth rate of the hydrodynamic mode. On the other hand, the growth rate of the magnetic mode remains bounded by the one of the mixed mode, and remains below the hydro's one, in the magneto-elliptical case. The previous results concern very small $\varepsilon$ and are obtained analytically : In addition to differences between magnetoprecessional and magneto-elliptic instabilities identified at small $\varepsilon$, differences between KBF and MBF begin to appear at increasing $\varepsilon$. This effect is shown in a synoptic way in figures $6(\eta=1)$ and $7(\eta=5)$, for the three cases, KBF, MBF and elliptical, for values of $\varepsilon$ up to 0.4 .

Of course we have chosen here an idealized model: Studying the linear stage of instabilities in an unbounded medium without viscous effects. Future work will concern the nonlinear stage and saturation of these instabilities by looking at the various mode couplings induced by the nonlinearities of the system: The convective terms in $\boldsymbol{u} \cdot \boldsymbol{\nabla} \boldsymbol{b}$ and $\boldsymbol{u} \cdot \boldsymbol{\nabla} \boldsymbol{u}$ and the magnetic ones in $\boldsymbol{b} \times \boldsymbol{u}$ and $\boldsymbol{b} \cdot \boldsymbol{\nabla} \boldsymbol{u}$. Pseudo-spectral DNS in deformed periodic boxes are a natural continuation of "RDT" studies, and we will use them to reintroduce strong nonlinearity and "volume" dissipation, but still ignoring physical confinement. Such future DNS studies will give a new insight to developed turbulence triggered by well identified linear instabilities, without artificial forcing.

\section{Appendices}

In the following appendices, we will use the asymptotic procedure given by Lebovitz \& Zweibel [35] to derive the results discussed in $\S 4$. 


\section{Construction of the Floquet multiplier matrix $M$ at first order of $\varepsilon$}

Assuming that, at sufficiently small $\varepsilon$, the instability emanating from the point $P\left(0, \chi_{0}\right)$ of the plane $(\varepsilon, \chi)$ is a wedge apex, so that (see e.g., $[35,36])$,

$$
\chi=\chi_{0}+\nu \varepsilon+\mathcal{O}\left(\varepsilon^{2}\right)
$$

Accordingly, for fixed $\eta$, the expansion of the Floquet multiplier matrix $\mathbf{M}$ (described by equation (38)) in Taylor series around $\varepsilon=0$ and $\chi=\chi_{0}$ takes the form

$$
\mathbf{M}(\varepsilon, \chi)=\mathbf{M}_{0}+\varepsilon \mathbf{M}_{1}+\mathcal{O}\left(\varepsilon^{2}\right)
$$

where

$$
\mathbf{M}_{0}=\mathbf{M}\left(0, \chi_{0}\right), \quad \mathbf{M}_{1}=\mathbf{M}_{\varepsilon}\left(0, \chi_{0}\right)+\nu \mathbf{M}_{\chi}\left(0, \chi_{0}\right)
$$

with the notation

$$
\mathbf{M}_{\varepsilon}=\frac{\partial \mathbf{M}}{\partial \varepsilon}, \quad \mathbf{M}_{\chi}=\frac{\partial \mathbf{M}}{\partial \chi}
$$

For the determination of the matrices $\mathbf{M}_{0}$ and $\mathbf{M}_{\varepsilon}$, we expand both the matrices $\mathbf{D}$ and $\boldsymbol{\Phi}$ in Taylor series around $\varepsilon=0$ at fixed $\chi$ and $\tau \in[0,2 \pi]$,

$$
\begin{aligned}
& \mathbf{D}(\tau, \varepsilon, \chi)=\mathbf{D}_{0}(\tau, \chi)+\varepsilon \mathbf{D}_{\varepsilon}(\tau, \chi)+\mathcal{O}\left(\varepsilon^{2}\right), \\
& \mathbf{\Phi}(\tau, \varepsilon, \chi)=\boldsymbol{\Phi}_{0}(\tau, \chi)+\varepsilon \boldsymbol{\Phi}_{1}(\tau, \chi)+\mathcal{O}\left(\varepsilon^{2}\right),
\end{aligned}
$$

with $\boldsymbol{\Phi}(0)=\mathbf{I}_{4}$ and $\boldsymbol{\Phi}_{1}(0)=\mathbf{0}$. The expression of $\mathbf{D}_{0}$ is given by equation (39), while the expression of $\mathbf{D}_{\varepsilon}$ can be deduced from the expansion (42),

$$
\begin{gathered}
\left(\mathbf{D}_{\varepsilon}\right)_{11}=2 \imath \varepsilon \chi \sqrt{1-\chi^{2}}\left(\mathrm{e}^{\imath \tau}-\mathrm{e}^{-\imath \tau}\right), \\
\left(\mathbf{D}_{\varepsilon}\right)_{12}=-\varepsilon \frac{\sqrt{1-\chi^{2}}}{\chi}\left(\mathrm{e}^{\imath \tau}+\mathrm{e}^{-\imath \tau}\right), \\
\left(\mathbf{D}_{\varepsilon}\right)_{21}=\varepsilon \chi \sqrt{1-\chi^{2}}\left(4 \chi^{2}-1\right)\left(\mathrm{e}^{\imath \tau}+\mathrm{e}^{-\imath \tau}\right),
\end{gathered}
$$

whereas the other components are zero. Accordingly, by substituting the form (64) into the Floquet system (38), we obtain

$$
\begin{gathered}
\frac{d \boldsymbol{\Phi}_{0}}{d \tau}=\mathbf{D}_{\mathbf{0}} \cdot \boldsymbol{\Phi}_{0}, \\
\frac{d \boldsymbol{\Phi}_{1}}{d \tau}=\mathbf{D}_{\mathbf{0}} \cdot \boldsymbol{\Phi}_{0}+\frac{\partial \mathbf{D}}{\partial \varepsilon} \cdot \boldsymbol{\Phi}_{0} .
\end{gathered}
$$


Because $\mathbf{D}_{0}$ is a constant matrix, as already indicated, the solution of the first differential system takes the form $\boldsymbol{\Phi}_{\mathbf{0}}=\exp \left(\tau \mathbf{D}_{0}\right)$ and hence $\mathbf{M}_{0}=\exp \left(2 \pi \mathbf{D}_{0}\right)$. On the other hand, by using the variation of constants formula, we may determine the solution of the second differential system in (66), which, at $\tau=2 \pi$, takes the form (see also $[35,36]$ ),

$$
\begin{gathered}
\mathbf{M}_{\varepsilon}(\chi)=\boldsymbol{\Phi}_{1}(2 \pi, \chi)=\left[\exp \left(2 \pi \mathbf{D}_{0}\right)\right] \mathbf{J}(\chi)= \\
{\left[\exp \left(2 \pi \mathbf{D}_{0}\right)\right]\left[\int_{0}^{2 \pi} \boldsymbol{\Phi}_{0}^{-1}(\tau, \chi) \mathbf{D}_{\varepsilon}(\tau, \chi) \boldsymbol{\Phi}_{0}(\tau, \chi) d \tau\right] .}
\end{gathered}
$$

To determine the eigenvalues of the Floquet multiplier matrix $\mathbf{M}$ it is simpler to work in the base diagonalizing the matrix $\mathbf{D}_{0}$.

\section{Calculations in the base diagonalizing $\mathbf{D}_{0}$}

The base diagonalizing $\mathbf{D}_{0}$ has been determined by Lebovitz \& Zweibel [35] and will repeated here for the sake of clarity. In the new basis, one has

$$
\tilde{\mathbf{D}}_{0}=\operatorname{diag}\left(\sigma_{1}, \sigma_{2}, \sigma_{3}, \sigma_{4}\right)=\mathbf{P}^{-1} \mathbf{D}_{0} \mathbf{P}
$$

where $\sigma_{i}(i=1,2,3,4)$ is described by equation (40) and

$$
\begin{gathered}
\mathbf{P}=\left[\begin{array}{cccc}
\sigma_{1} & \sigma_{2} & \sigma_{3} & \sigma_{4} \\
-\imath \chi \sigma_{1} & \imath \chi \sigma_{2} & -\imath \chi \sigma_{3} & \imath \chi \sigma_{4} \\
\imath \eta \chi & \imath \eta \chi & \imath \eta \chi & \imath \eta \chi \\
\eta \chi^{2} & -\eta \chi^{2} & \eta \chi^{2} & -\eta \chi^{2}
\end{array}\right] \\
\mathbf{P}^{-1}=\frac{1}{4 \chi^{2} \eta \sqrt{1+\eta^{2}}}\left[\begin{array}{cccc}
-\imath \chi \eta & \eta & \sigma_{3} & \imath \sigma_{3} / \chi \\
\imath \chi \eta & \eta & \sigma_{3} & -\imath \sigma_{3} / \chi \\
\imath \chi \eta & -\eta & -\sigma_{1} & -\imath \sigma_{1} / \chi \\
-\imath \chi \eta & -\eta & -\sigma_{1} & \imath \sigma_{1} / \chi
\end{array}\right] .
\end{gathered}
$$

It follows that, in the new basis, the transformed matrix $\tilde{\mathbf{M}}_{\varepsilon}=\mathbf{P}^{-1} \mathbf{M}_{\varepsilon} \mathbf{P}$ takes the form

$$
\begin{gathered}
\tilde{\mathbf{M}}_{\varepsilon}(\chi)=\tilde{\mathbf{M}}_{0}(\chi) \tilde{\mathbf{J}}(\chi)= \\
\tilde{\mathbf{M}}_{0}(\chi)\left[\int_{0}^{2 \pi} \tilde{\boldsymbol{\Phi}}_{0}^{-1}(\tau, \chi) \tilde{\mathbf{D}}_{\varepsilon}(\tau, \chi) \tilde{\boldsymbol{\Phi}}_{0}(\tau, \chi) d \tau\right]
\end{gathered}
$$


where $\tilde{\mathbf{M}}_{0}(\chi)=\exp \left(2 \pi \tilde{\mathbf{D}}_{0}\right)$ because the eigenvalues $\sigma_{i}(i=1,2,3,4)$ are distinct (see equation (40)). For the KBF case (ot the MBF case) the matrix $\mathbf{D}_{\varepsilon}$ has only three non-zero components, those given by (66). Hence, the component $\tilde{J}_{i j}$ can be written as

$$
\tilde{\mathbf{J}}_{i j}=P_{i 1}^{-1} H_{11} P_{1 j}+P_{i 1}^{-1} H_{12} P_{2 j}+P_{i 2}^{-1} H_{21} P_{1 j}
$$

where

$$
\begin{aligned}
& H_{11}=\int_{0}^{2 \pi} \mathrm{e}^{\left(\sigma_{j}-\sigma_{i}\right) \tau}\left(\mathbf{D}_{\varepsilon}\right)_{11}(\tau) d \tau, \\
& H_{12}=\int_{0}^{2 \pi} \mathrm{e}^{\left(\sigma_{j}-\sigma_{i}\right) \tau}\left(\mathbf{D}_{\varepsilon}\right)_{12}(\tau) d \tau, \\
& H_{21}=\int_{0}^{2 \pi} \mathrm{e}^{\left(\sigma_{j}-\sigma_{i}\right) \tau}\left(\mathbf{D}_{\varepsilon}\right)_{21}(\tau) d \tau .
\end{aligned}
$$

The above integrals vanish when $\left(\sigma_{j}-\sigma_{i}\right) \neq \pm \imath$, or equivalently, when $\left(\omega_{j}-\omega_{i}\right) \neq \pm 1$. Otherwise, one finds

$$
H_{12}=-2 \pi \frac{\sqrt{1-\chi^{2}}}{\chi}, \quad H_{21}=2 \pi \chi\left(4 \chi^{2}-1\right) \sqrt{1-\chi^{2}}
$$

for both $\left(\omega_{j}-\omega_{i}\right)= \pm 1$, and

$$
H_{11}= \pm 4 \imath \pi \chi \sqrt{1-\chi^{2}} \text { for } \quad\left(\omega_{j}-\omega_{i}\right)=\mp 1
$$

respectively. This implies that the diagonal components of the matrix $\tilde{\mathbf{J}}$ are zero, i.e., $\tilde{J}_{j j}=0$. For convenience, we give here only the expression of the off-diagonal elements $\tilde{J}_{i j}$ that will be used later,

$$
\begin{gathered}
\omega_{1} \tilde{J}_{12}=\omega_{2} \tilde{J}_{21}=\frac{\imath \pi \omega_{1} \omega_{2}}{\chi \sqrt{1+\eta^{2}}} \sqrt{1-\chi^{2}}\left|2 \chi^{2}+\chi-1\right| \\
\omega_{1} \tilde{J}_{13}=\omega_{3} \tilde{J}_{31}=\frac{\imath \pi \omega_{1} \omega_{3}}{\sqrt{1+\eta^{2}}} \sqrt{1-\chi^{2}}(2 \chi+1) \\
\omega_{3} \tilde{J}_{34}=\omega_{4} \tilde{J}_{43}=\frac{\imath \pi \omega_{3} \omega_{4}}{\chi \sqrt{1+\eta^{2}}} \sqrt{1-\chi^{2}}\left|-2 \chi^{2}+\chi+1\right| \\
-\omega_{4} \tilde{J}_{41}=\omega_{1} \tilde{J}_{14}=\frac{\imath \pi \omega_{1} \omega_{4}}{\chi \sqrt{1+\eta^{2}}} \sqrt{1-\chi^{2}}\left|2 \chi^{2}+\chi-1\right| .
\end{gathered}
$$

We note that, for the elliptical flow case studied by [35], it is found that $\tilde{J}_{j j} \neq 0$ (see equation $\mathrm{A}(26)$ in $[35])$. 
To determine the transformed matrix $\tilde{\mathbf{M}}_{1}=\mathbf{P}^{-1} \mathbf{M}_{1} \mathbf{P}$ where $\mathbf{M}_{1}$ appears in equation (63), we need the derivative

$$
\tilde{\mathbf{M}}_{\chi}(0, \chi)=\frac{\partial \tilde{\mathbf{M}}(0, \chi)}{\partial \chi}=\frac{\partial \tilde{\mathbf{M}}_{0}(\chi)}{\partial \chi}
$$

Due to the fact that

$$
\tilde{\mathbf{M}}_{0}=\operatorname{diag}\left[\exp \left(2 \pi \sigma_{1}\right), \ldots, \exp \left(2 \pi \sigma_{4}\right)\right]
$$

and $\left(\partial \sigma_{i} / \partial \chi\right)=\sigma_{i} / \chi$ (see equation (40)), we deduce that

$$
\tilde{\mathbf{M}}_{\chi}(0, \chi)=\frac{2 \pi}{\chi} \operatorname{diag}\left[\sigma_{1} \exp \left(2 \pi \sigma_{1}\right), \ldots, \sigma_{4} \exp \left(2 \pi \sigma_{4}\right)\right] .
$$

It follows that

$$
\begin{gathered}
\left(\tilde{\mathbf{M}}_{1}\right)_{j j}=\frac{2 \nu \pi}{\chi} \sigma_{j} \exp \left(2 \pi \sigma_{j}\right), \quad(j=1,2,3,4) \\
\left(\tilde{\mathbf{M}}_{1}\right)_{i j}=\left[\exp \left(2 \pi \sigma_{i}\right)\right] \tilde{J}_{i j} \quad(i \neq j) .
\end{gathered}
$$

\section{Characteristic polynomial}

We expand the characteristic polynomial

$$
p(\varepsilon, \chi)=\left|\mathbf{M}(\varepsilon)-\lambda I_{4}\right|
$$

in perturbation series around $\varepsilon=0$,

$$
p(\lambda, \varepsilon)=p_{0}(\lambda)+\varepsilon p_{1}(\lambda)+\varepsilon^{2} p_{2}(\lambda)+\mathcal{O}\left(\varepsilon^{3}\right)
$$

where, $p_{0}(\lambda)=\Pi_{i=1}^{4}\left(\lambda_{i}-\lambda\right)=\Pi_{i=1}^{4}\left(\exp \left(2 \pi \sigma_{i}\right)-\lambda\right)$ is the characteristic polynomial of the matrix $\tilde{\mathbf{M}}_{0}$. The roots of $p(\lambda, \varepsilon)$ are denoted by $\Lambda_{1}, \Lambda_{2}, \ldots$

The condition for destabilization is that there exist a double (or higher) roots of $p(\lambda, \varepsilon)$. For instance, assuming that $\lambda_{1}=\lambda_{2}$ and expanding the root $\Lambda_{1}$ in the form of a Puiseux expansion (see e.g. Hille [40]),

$$
\Lambda_{1}=\lambda_{1}+\varepsilon^{1 / 2} \beta_{1 / 2}+\varepsilon \beta_{1}+\mathcal{O}\left(\varepsilon^{3 / 2}\right)
$$

Because $p_{1}\left(\lambda_{1}\right)=0$, one shows that $\beta_{1 / 2}=0$ and $\beta_{1}$ is a solution of the second-order algebraic equation (see Appendices A3 and B in [35]),

$$
a_{0} \beta_{1}^{2}+a_{1} \beta_{1}+a_{2}=0,
$$


with

$$
\begin{gathered}
a_{0}=\frac{1}{2}\left[\frac{d^{2} p_{0}}{d \lambda^{2}}\right]_{\lambda=\lambda_{1}}=\left(\lambda_{3}-\lambda_{1}\right)\left(\lambda_{4}-\lambda_{1}\right), \\
a_{1}=\left[\frac{d p_{0}}{d \lambda}\right]_{\lambda=\lambda_{1}}=-\left[\left(\tilde{\mathbf{M}}_{1}\right)_{11}+\left(\tilde{\mathbf{M}}_{1}\right)_{22}\right] a_{0}, \\
a_{2}=p_{2}\left(\lambda_{1}\right)=\left|\begin{array}{ll}
\left(\tilde{\mathbf{M}}_{1}\right)_{11} & \left(\tilde{\mathbf{M}}_{1}\right)_{12} \\
\left(\tilde{\mathbf{M}}_{1}\right)_{21} & \left(\tilde{\mathbf{M}}_{1}\right)_{22}
\end{array}\right| a_{0} .
\end{gathered}
$$

By setting $\gamma=\beta_{1} / \lambda_{1}$, equation (76) is rewritten as

$$
\frac{\Lambda_{1}}{\lambda_{1}}=1+\varepsilon \gamma+\mathcal{O}\left(\varepsilon^{3 / 2}\right), \quad \gamma=\frac{1}{2 a_{0}}\left[a_{1} \pm \sqrt{a_{1}^{2}-4 a_{0} a_{2}}\right]
$$

Consequently, at first order of $\varepsilon$, there is instability if $\Re \gamma \neq 0$. For the cases where $\lambda_{1}=\lambda_{3}$, $\lambda_{3}=\lambda_{4}$ or $\lambda_{1}=\lambda_{4}$, the coefficients $a_{0}, a_{1}$ and $a_{2}$ are calculated in a similar manner.

\section{Maximal growth rate and width of the subharmonic instabilities}

As noted in $\S 4$, the "hydrodynamic" modes are the modes for which $\lambda_{1}=\lambda_{2}$. The substitution of (73) into (74) allows us to determine the coefficients $a_{1}, a_{1}$ and $a_{2}$. Because, in that case one has $a_{1}=0$, the coefficient $\gamma$ (given by (78)) takes the form

$$
\gamma^{2}=\frac{\pi^{2}}{\chi^{2}}\left[-\nu^{2}+\frac{\left(1-\chi^{2}\right)}{4 \chi^{2}\left(1+\eta^{2}\right)}\left(2 \chi^{2}+\chi-1\right)^{2}\right] \text {. }
$$

Both the maximal growth rate $\sigma_{\max }$ and the width $\delta$ of the subharmonic "hydrodynamic" instability can be deduced from the above relation,

$$
\begin{gathered}
\frac{\sigma_{\max }}{\varepsilon}=\frac{\max (\Re \gamma)}{2 \pi}, \\
\delta=\left(\nu_{+}-\nu_{-}\right) \varepsilon,
\end{gathered}
$$

such that $\nu_{+}$and $\nu_{-}$are the roots of the algebraic equation $\Re \gamma(\nu)=0$. It follows that

$$
\frac{\sigma_{\max }}{\varepsilon}=\frac{\sqrt{1-\chi^{2}}}{4 \chi \sqrt{1+\eta^{2}}}\left|2 \chi^{2}+\chi-1\right|,
$$

and

$$
\nu^{2}=\frac{\left(1-\chi^{2}\right)}{4 \chi^{2}\left(1+\eta^{2}\right)}\left(2 \chi^{2}+\chi-1\right)^{2}
$$

or equivalently,

$$
\nu_{+}=-\nu_{-}=\frac{\sqrt{1-\chi^{2}}}{2 \sqrt{1+\eta^{2}}}\left|2 \chi^{2}+\chi-1\right| .
$$


In the case with $\lambda_{1}=\lambda_{3}$ (i.e., the so called "mixed modes"), the coefficients $a_{0}, a_{1}$ and $a_{2}$ in (77) take the form

$$
\begin{gathered}
a_{0}=\frac{1}{2}\left[\frac{d^{2} p_{0}}{d \lambda^{2}}\right]_{\lambda=\lambda_{1}}=\left(\lambda_{2}-\lambda_{1}\right)\left(\lambda_{4}-\lambda_{1}\right), \\
a_{1}=\left[\frac{d p_{0}}{d \lambda}\right]_{\lambda=\lambda_{1}}=-\left[\left(\tilde{\mathbf{M}}_{1}\right)_{11}+\left(\tilde{\mathbf{M}}_{1}\right)_{33}\right] a_{0}, \\
a_{2}=p_{2}\left(\lambda_{1}\right)=\left|\begin{array}{ll}
\left(\tilde{\mathbf{M}}_{1}\right)_{11} & \left(\tilde{\mathbf{M}}_{1}\right)_{13} \\
\left(\tilde{\mathbf{M}}_{1}\right)_{31} & \left(\tilde{\mathbf{M}}_{1}\right)_{33}
\end{array}\right| a_{0} .
\end{gathered}
$$

Similarly, the substitution of (73) into (74) allows us to calculate these coefficients and determine the coefficient $\gamma$,

$$
\gamma=2 \imath \pi \nu \pm \sqrt{D}, \quad D=\frac{\pi^{2}\left(1-4 \chi^{2}\right)^{2}\left(1-\chi^{2}\right)}{4\left(1+\eta^{2}\right)}-\frac{\pi^{2} \nu^{2}}{\chi^{2}}
$$

It appears that $\Re \gamma$ is maximal for $\nu=0$, so that,

$$
\frac{\sigma_{\max }}{\varepsilon}=\frac{\sqrt{1-\chi^{2}}}{4 \chi \sqrt{1+\eta^{2}}}\left|4 \chi^{2}-1\right|
$$

while the slopes $\nu_{-}$and $\nu_{+}$, that are the roots of the algebraic equation $\Re \gamma(\nu)=D=0$, take the form

$$
\nu_{+}=-\nu_{-}=\frac{\chi \sqrt{1-\chi^{2}}}{2 \sqrt{1+\eta^{2}}}\left|1-4 \chi^{2}\right| .
$$

Similarly, for the case where $\lambda_{3}=\lambda_{4}$, which characterizes the subharmonic "magnetic" modes, we determine the expression of the coefficient $\gamma$,

$$
\gamma^{2}=\frac{\pi^{2}}{\chi^{2}}\left[\frac{\left(1-\chi^{2}\right)\left(1+\chi-2 \chi^{2}\right)^{2}}{4\left(1+\eta^{2}\right)}-\nu^{2}\right]
$$

from which we deduce

$$
\begin{gathered}
\frac{\sigma_{\max }}{\varepsilon}=\frac{\sqrt{1-\chi^{2}}}{4 \chi \sqrt{1+\eta^{2}}}\left|1+\chi-2 \chi^{2}\right|, \\
\nu_{-}=-\nu_{+}=\frac{\sqrt{1-\chi^{2}}}{2 \sqrt{1+\eta^{2}}}\left|1+\chi-2 \chi^{2}\right| .
\end{gathered}
$$

We finally consider the case where $\lambda_{1}-\lambda_{4}=1$. In that case, one has $\chi=1 / 2$, which gives $\tilde{J}_{14}=\tilde{J}_{41}=0$ (see equation $\left.(73)\right)$. Accordingly, one shows that the coefficient $\gamma$ is purely imaginary,

$$
\gamma=2 \imath \pi \nu\left(\sqrt{1+\eta^{2}} \pm 1\right)
$$

meaning that, when $\lambda_{1}=\lambda_{4}$, there is no instability. 
An alternative way which better reflects the physical processes especially for rotating flows is to use a local frame in which both the geometrical constraints $\boldsymbol{k} \cdot \hat{\boldsymbol{u}}=0$ and $\boldsymbol{k} \cdot \hat{\boldsymbol{b}}=0$ are satisfied by construction (see e.g. [38]). The orthonormal basis of this frame is defined as

$$
\boldsymbol{e}^{(1)}=\boldsymbol{k} \times \boldsymbol{n} /\|\boldsymbol{k} \times \boldsymbol{n}\|, \quad \boldsymbol{e}^{(2)}=\boldsymbol{k} \times \boldsymbol{e}^{(1)} / k, \quad \boldsymbol{e}^{(3)}=\boldsymbol{k} / k,
$$

or equivalently, by considering that the unit vector $\boldsymbol{n}$ aligns with the solid rotation axis (i.e., $\left.\boldsymbol{n}=\boldsymbol{e}_{3}\right)$

$$
\boldsymbol{e}^{(1)}=\left[\frac{k_{2}}{k_{h}},-\frac{k_{1}}{k_{h}}, 0\right]^{T}, \quad \boldsymbol{e}^{(2)}=\left[\frac{k_{1}}{k_{h}} \frac{k_{3}}{k}, \frac{k_{2}}{k_{h}} \frac{k_{3}}{k}, \quad-\frac{k_{h}}{k}\right]^{T} .
$$

where $k_{h}=\sqrt{k_{1}^{2}+k_{2}^{2}}$ is the horizontal wave number In that frame, $\hat{\boldsymbol{u}}$ (respectively $\hat{\boldsymbol{b}}$ ) has only two components,

$$
\hat{u}_{i}=e_{i}^{(1)} u^{(1)}+e_{i}^{(2)} u^{(2)}, \quad \hat{b}_{i}=e_{i}^{(1)} b^{(1)}+e_{i}^{(2)} b^{(2)}, \quad(i=1,2,3) .
$$

where $u^{(1)}$ and $u^{(2)}$ are subsequently coined "toroidal" and "poloidal" in reference to their meaning in physical space [38], and they are related to the variables $\left(c_{1}, c_{2}, c_{3}, c_{4}\right)$ as

$$
\begin{gathered}
\left(c_{1}, c_{3}\right)=-k_{h}\left(u^{(1)}, b^{(1)}\right)-2 \varepsilon \frac{k_{h} k_{2}}{k}\left(u^{(2)}, b^{(2)}\right), \\
\left(c_{2}, c_{4}\right)=\frac{k_{h} k_{0}}{k}\left(u^{(2)}, b^{(2)}\right)
\end{gathered}
$$

for the KBF case, and

$$
\begin{aligned}
& \left(c_{1}, c_{3}\right)=-\left(k_{h}+2 \varepsilon \frac{k_{1} k_{0}}{k_{h}^{2}}\right)\left(u^{(1)}, b^{(1)}\right)+2 \varepsilon \frac{k_{2} k_{0}^{2}}{k k_{h}}\left(u^{(2)}, b^{(2)}\right), \\
& \left(c_{2}, c_{4}\right)=2 \varepsilon \frac{k_{2} k_{0}}{k_{h}}\left(u^{(1)}, b^{(1)}\right)+\left(2 \varepsilon \frac{k_{1} k_{0}^{2}}{k k_{h}}+\frac{k_{0} k_{h}}{k}\right)\left(u^{(2)}, b^{(2)}\right)
\end{aligned}
$$

for the MBF case. In the local frame, the Floquet system for the poloidal and toroidal modes takes the form

$$
\frac{d}{d \tau}\left[\begin{array}{c}
u^{(1)} \\
u^{(2)} \\
b^{(1)} \\
b^{(2)}
\end{array}\right]=\left[\begin{array}{cccc}
0 & m_{12} & \imath \chi \eta & 0 \\
m_{21} & m_{22} & 0 & \imath \chi \eta \\
\imath \chi \eta & 0 & 0 & m_{34} \\
0 & \imath \chi \eta & m_{43} & -m_{22}
\end{array}\right] \cdot\left[\begin{array}{c}
u^{(1)} \\
u^{(2)} \\
b^{(1)} \\
b^{(2)}
\end{array}\right]
$$


with

$$
\begin{gathered}
m_{12}=2 \frac{k_{0}}{k}, \quad m_{21}=-2 \frac{\left(k_{0}-\varepsilon k_{1}\right)}{k}, \quad m_{22}=-2 \varepsilon \frac{k_{2} k_{3}}{k^{2}}, \\
m_{34}=m_{21}, \quad m_{43}=2 \frac{\left(k_{0}-2 \varepsilon k_{1}\right)}{k}
\end{gathered}
$$

for the $\mathrm{KBF}$ case and

$$
\begin{gathered}
m_{12}=2 \frac{k_{0}}{k}+2 \varepsilon \frac{k_{1} k}{k_{h}^{2}}, \quad m_{21}=-2 \frac{\left(k_{0}-\varepsilon k_{1}\right)}{k}-2 \varepsilon \frac{k_{1} k}{k_{h}^{2}}, \quad m_{22}=-2 \varepsilon \frac{k_{2} k_{3}}{k^{2}}, \\
m_{34}=-2 \varepsilon \frac{k_{1}}{k}+2 \varepsilon \frac{k_{1} k}{k_{h}^{2}}, \quad m_{43}=-2 \varepsilon \frac{k_{1} k}{k_{h}^{2}},
\end{gathered}
$$

for the MBF case.

[1] S. C. Crow, AIAA J., 8, 2172 (1970).

[2] R.T. Pierrehumbert, Phys. Rev. Lett. 57, 2157 (1986).

[3] B.J. Bayly, Phys. Rev. Lett. 57, 2160 (1986).

[4] R.R. Kerswell, Annu. Rev. Fluid Mech., 34, 83 (2002).

[5] G. K. Batchelor and I. Proudman, Q. J. Mech. Appl. Math. 7, 83 (1954).

[6] H. K. Moffatt, Colloquium On Atmospheric Turbulence and Radio Wave Propagation, edited by A. M. Yaglom and V. I. Tatarski (Nauka, Moscow), 139 (1967).

[7] C. Cambon, J. -P. Benoit, L. Shao, and L. Jacquin, J. Fluid Mech., 278, 175 (1994).

[8] C. H. Eloy, P. Le Gal and S. Le Dizès, Phys. Rev. Lett. 85, 3400 (2000).

[9] C. H. Eloy, P. Le Gal and S. Le Dizès, J. Fluid Mech., 476, 357 (2003).

[10] L. Lacaze, W. Herreman, M. Le Bars, S. Le Dizès and P. Le Gal, Geophys. Astrophys. Fluid Dynamics, , 100, 299 (2006).

[11] R. R. Kerswell, Geophys. Astrophys. Fluid Dynamics, 72, 107 (1993).

[12] A. Mahalov, Phys. Fluids A 5, 891 (1993).

[13] A. Salhi and C. Cambon, Phys. Rev. E, 79, 036303 (2009).

[14] R. F. Gans, J. Fluid Mech., 41-4, 865 (1970).

[15] P. Meunier, C. Eloy, R. Lagrange and F. Nadal, J. Fluid Mech., 599, 405 (2008).

[16] T. Lehner, W. Mouhali, J. Léorat and A. Mahalov, Geophys. Astrophys. Fluid Dynamics, to appear.

[17] B. Lehnert, Astrophys. J., 119, 647 (1954). 
[18] B. Lehnert, Quart. Appl. Math., 4, 321 (1955).

[19] S. Chandrasekhar, Hydrodynamic and hydromagnetic stability, Clarendon Press, Oxford (1961).

[20] S. I. Braginsky, Geophys. Astro. Fluid Dyn., 14, 189 (1979).

[21] S. I. Braginsky, Geomagn. Aeron., 4, 698 (1964).

[22] S. A. Balbus and J. F. Hawley, Rev. Mod. Phys. 70, 1 (1998).

[23] I. V. Khalzov et al., Phys. Fluids, 18, 124107 (2006).

[24] B. Jamroz, K. Julien and E. Knobloch, Phys. Scr., 132, 014027 (2008a).

[25] B. Jamroz, K. Julien and E. Knobloch, A N, 329, 675 (2008b).

[26] R. F. Gans, J. Fluid Mech., 45-1, 111 (1970).

[27] E. C. Bullard, The Magnetic Field within the Earth, Proc. of the Royal Society of London. Series A, Mathematical and Physical Sciences, 197, 433 (1949).

[28] W. V. Malkus, Science, 160 (3825), 259, (1968).

[29] Zhang, K., Liao, X., and Li, L. (2003), Celestial Mech. Dyn. Astronomy, 87, 39 (2003).

[30] A. Tilgner, Phys. Fluids, 17(3), 034104 (2005).

[31] C. Wu and P. H. Roberts, Geophys. Astrophys. Fluid Dynamics, 103, 467 (2009).

[32] A. Gailitis, O. Lielausis, E. Platacis, G. Gerbeth and F. Stefani, Reviews of Modern Physics, 74, 973 (2002).

[33] A.D.D. Craik, J. Fluid Mech., 198, 275 (1989).

[34] F. Waleffe, Phys. Fluids 2, 76 (1990).

[35] N. R. Lebovitz and E. Zweibel, Astrophys. J., 609, 301, (2004).

[36] K.A. Mizerski and K. Bajer, J. Fluid Mech., 632, 401 (2009).

[37] R. J. Wiener, P. W. Hammer, C. E. Swanson \& R. J. Donnelly, Phys. Rev. Lett. 64, 1115 (1990).

[38] P. Sagaut \& C. Cambon, Homogeneous turbulence dynamics, Cambridge University Press (2008).

[39] C. M. Bender \& S. A. Orszag, Advanced mathematical methods for scientists and engineers, Mc Graw-Hill (1978).

[40] E. Hille, Analytic function theory, Volume 2 (New York: Ginn) (1962).

[41] V. A. Yakubovich and V. M. Starzhinsky, Linear differential equations with periodic coeffcients. (New York: Wiley) (1975). 
[42] A. Salhi and C. Cambon, Phys. Rev. E, 81, 026302 (2010). 